\title{
Shape measures of random increasing $k$-trees*
}

\author{
Alexis Darrasse ${ }^{\dagger}$ \\ APR - LIP6 \\ UPMC \\ 75005 Paris France \\ alexis.darrasse@lip6.fr \\ Hsien-Kuei Hwang \\ Institute of Statistical Science \\ Academia Sinica \\ Taipei 115 Taiwan \\ hkhwang@stat.sinica.edu.tw \\ Michèle Soria $^{\dagger}$ \\ APR - LIP6 \\ UPMC \\ 75005 Paris France \\ michele.soria@lip6.fr
}

August 25, 2011

\begin{abstract}
Random increasing $k$-trees represent an interesting, useful class of strongly dependent graphs that have been studied widely, including being used recently as models for complex networks. We study in this paper an informative notion called connectivity-profile and derive, by several analytic means, asymptotic estimates for its expected value, together with the limiting distribution in certain cases; some interesting consequences predicting more precisely the shapes of random $k$-trees are also given. Our methods of proof rely essentially on a bijection between $k$-trees and ordinary trees, and the resolution of a linear system.
\end{abstract}

Key words. Random graphs, $k$-trees, differential equations, limiting distribution, generating functions, connectivity-profile, height, width, singularity analysis, random generation, method of moments, Flajolet-Odlyzko admissible functions.

\section{Contents}

1 Introduction 1

2 Random increasing $k$-trees and generating functions 5

2.1 Increasing $k$-trees and the bijection. . . . . . . . . . . . . 5

2.2 Generating functions. . . . . . . . . . . . . . . . . 6

*An extended abstract of this paper appeared in [14].

${ }^{\dagger}$ This work was partially supported by ANR under the contract MAGNUM, n²010 BLAN 0204. 
3 Expected connectivity-profile $\quad 8$

3.1 The expected value . . . . . . . . . . . . . . . . . 8

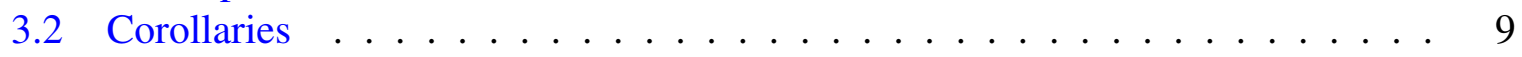

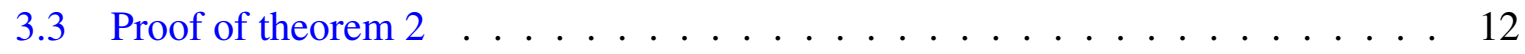

3.3.1 Bivariate generating function and the linear system . . . . . . . . 12

3.3.2 Converting the linear system (13) into scalar DEs . . . . . . . . . . . 14

3.3.3 Solution to the Cauchy-Euler DEs (14) . . . . . . . . . . . . . 15

3.3.4 Properties of the zeros of $P_{k}$ and local expansions of $\lambda_{1}(w) \ldots \ldots$

3.3.5 From $\mathscr{M}_{j}$ to $\tilde{M}_{d, j} \ldots \ldots \ldots \ldots \ldots$

3.4 Proofs of some corollaries of Theorem 2 . . . . . . . . . . . . . . . 21

4 Limiting distributions

4.1 Distribution of the connectivity-profile . . . . . . . . . . . . 23

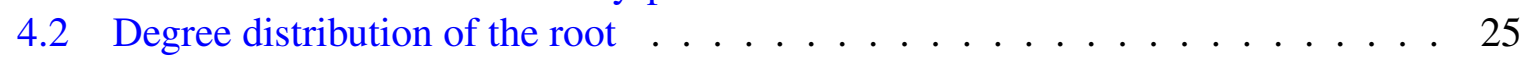

4.3 Limiting distribution: $d \geq 1$ and $1 \leq j \leq k \ldots \ldots \ldots$

4.3 .1 Recurrence relations . . . . . . . . . . . . . . . 28

4.3.2 Flajolet-Odlyzko admissible functions . . . . . . . . . . . . . . . . 29

4.3.3 Method of moments . . . . . . . . . . . . . . . 30

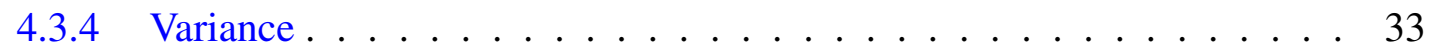

$5 \quad$ Generation of random $k$-trees $\quad 34$

\section{Introduction}

A $k$-tree is a graph reducible to a $k$-clique by successive removals of a vertex of degree $k$ whose neighbors form a $k$-clique. This class of $k$-trees has been widely studied in combinatorics (for enumeration and characteristic properties $[5,48]$ ), in graph algorithms (many NP-complete problems on graphs can be solved in polynomial time on $k$-trees [2]), and in many other fields where $k$-trees were naturally encountered (see [2]). By construction, vertices in such structures are remarkably close, reflecting a highly strongly dependent graph structure, and they exhibit with no surprise the scale-free property [28], yet somewhat unexpectedly many properties of random $k$-trees can be dealt with by standard combinatorial, asymptotic and probabilistic tools, thus providing an important model of synergistic balance between mathematical tractability and the predictive power for practical-world complex networks.

While the term " $k$-trees" is not very informative and may indeed be misleading to some extent, the combinatorial object stands out by their underlying recursive tree structure, which facilitates the analysis of the properties and the exploration of the structure. Indeed, for $k=1$, $k$-trees are just ordinary trees, and for $k \geq 2$ a bijection [15] between $k$-trees and a nontrivial simple family of trees can be explicitly defined, which we will describe briefly in this paper.

To reduce ambiguity of terms, except for "k-trees", all other occurrences of "trees" in this paper are ordinary trees without cycles. Also the term " $k$-tree" has been used in several other areas with very different meanings; see for example [7, 23, 35, 37, 40, 45, 52].

The process of generating a $k$-tree begins with a $k$-clique, which is itself a $k$-tree; then the $k$-tree grows by linking a new vertex to every vertex of an existing $k$-clique, and to these vertices only. The same process continues; see Figure 1 for an illustration. Such a simple construction process is reminiscent of several other structures proposed in the literature such as 


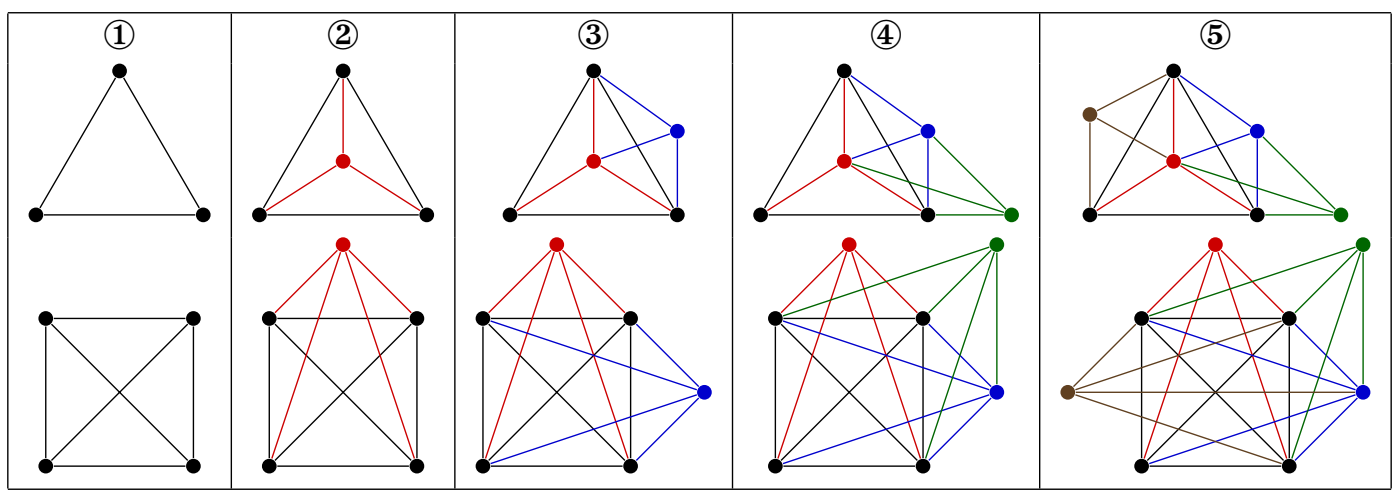

Figure 1: The first few steps of generating a 3-tree (top) and a 4-tree (bottom). Obviously, these graphs show the high connectivity of $k$-trees.

$k$-DAGs [17], random circuits [3], preferential attachment [4, 9, 32], and many other models (see, for example, $[8,22,41]$ ). While the construction rule in each of these models is very similar, namely, linking a new vertex to $k$ existing ones, the mechanism of choosing the existing $k$ vertices differs from one case to another, resulting in very different topology and dynamics.

Different variants of $k$-trees have been proposed in the literature depending on the modeling needs and they differ mainly in the procedure of choosing a $k$-clique each time a new vertex is added. So $k$-trees can be either labeled [5], unlabeled [34], increasing [54], planar [54], non-planar [5], ordered [43], or plane [42] (planar with a given embedding in the plane), etc.

For example, the family of random Apollonian networks, corresponding to planar 3-trees, has recently been employed as a model for complex networks [1, 54]. In these frameworks, since the exact topology of the real networks is difficult or even impossible to describe, one is often led to the study of models that present similarities to some observed properties such as the degree of a node and the distance between two nodes of the real structures.

For the purpose of this paper, we distinguish between two models of random labeled nonplane $k$-trees; by non-plane we mean that we consider these graphs as given by a set of edges (and not by its graphical representation):

- random simply-generated $k$-trees, which correspond to a uniform probability distribution on this class of labeled $k$-trees, and

- random increasing $k$-trees, where we consider the iterative generation process: at each time step, all existing $k$-cliques are equally likely to be selected and the new vertex is added with a label that is greater than the existing ones.

The two models are in good analogy to the simply-generated family of trees of Meir and Moon [38] marked specially by the functional equation $f(z)=z \Phi(f(z))$ for the underlying enumerating generating function, and the increasing family of trees of Bergeron et al. [6], characterized by the differential equation (abbreviated throughout as $D E) f^{\prime}(z)=\Phi(f(z))$. Very different stochastic behaviors have been observed for these families of trees. While similar in structure to these trees, the analytic problems on random $k$-trees we are dealing with here are however more involved because instead of a single scalar equation (either functional, algebraic, or differential), we now have a system of equations. 


\begin{tabular}{|r||c|c|}
\hline \multicolumn{1}{|c||}{ Model } & Simply-generated structures & Increasing structures \\
\hline Combinatorial description & $\mathcal{T}_{s}=\operatorname{Set}\left(\mathcal{Z} \times \mathcal{T}_{s}^{k}\right)$ & $\mathcal{T}=\operatorname{Set}\left(\mathcal{Z}^{\square} \times \mathcal{T}^{k}\right)$ \\
\hline Generating function & $T_{s}(z)=\exp \left(z T_{s}^{k}(z)\right)$ & $T^{\prime}(z)=T^{k+1}(z)$ \\
\hline Expansion near singularity & $T_{s}(z)=\tau-h \sqrt{1-z / \rho}+\ldots$ & $T(z)=(1-k z)^{-1 / k}$ \\
\hline Mean distance of nodes & $O(\sqrt{n})$ & $O(\log n)$ \\
\hline Degree distribution & Power law with exp. tails & Power law [28] \\
\hline Root-degree distribution & Power law with exp. tails & Stable law (Theorem 3) \\
\hline Expected Profile & Rayleigh limit law & Gaussian limit law (10) \\
\hline
\end{tabular}

Table 1: The contrast of some properties between random simply-generated $k$-trees and random increasing $k$-trees. Here $\mathcal{Z}$ denotes a node and $\mathcal{Z}^{\square}$ means a node with the smallest label.

It is known that random trees in the family of increasing trees are often less skewed, less slanted in shape, a typical description being the logarithmic order for the distance of two randomly chosen nodes; this is in sharp contrast to the square-root order for random trees belonging to the simply-generated family; see for example [6, 18, 27, 36, 38]. Such a contrast has inspired and stimulated much recent research. Indeed, the majority of random trees in the literature of discrete probability, analysis of algorithms, and random combinatorial structures are either $\log n$-trees or $\sqrt{n}$-trees, $n$ being the tree size. While the class of $\sqrt{n}$-trees have been extensively investigated by probabilists and combinatorialists, $\log n$-trees are comparatively less addressed, partly because most of them were encountered not in probability or in combinatorics, but in the analysis of algorithms. The class of random $k$-trees we addressed here, closer in property to $\log n$-trees, issues from the study of complex networks.

Table 1 presents a comparison of the two models: the classes $\mathcal{T}_{s}$ and $\mathcal{T}$, corresponding respectively to simply-generated $k$-trees and increasing $k$-trees. The results concerning simple $k$-trees are given in $[15,16]$, and those concerning increasing $k$-trees are derived in this paper (except for the power law distribution [28]). We start with the specification, described in terms of operators of the symbolic method [24]. A structure of $\mathcal{T}_{s}$ is a set of $k$ structures of the same type, whose roots are attached to a new node: $\mathcal{T}_{s}=\operatorname{Set}\left(\mathcal{Z} \times \mathcal{T}_{s}^{k}\right)$, while a structure of $\mathcal{T}$ is an increasing structure, in the sense that the new nodes get labels that are greater than those of the underlying structure (this constraint is reflected by the box-operator) $\mathcal{T}=\operatorname{Set}\left(\mathcal{Z}^{\square} \times \mathcal{T}^{k}\right)$. The analytic difference immediately appears in the enumerative generating functions that translate the specifications: the simply-generated structures are defined by $T_{s}(z)=\exp \left(z T_{s}^{k}(z)\right)$ and the corresponding increasing structures satisfy the $\mathrm{DE} T^{\prime}(z)=T^{k+1}(z)$. These equations lead to a singular expansion of the square-root type in the simply-generated model, and a singular expansion in terms of $(1-k z)^{-1 / k}$ in the increasing model. Similar analytic differences arise in the bivariate generating functions of shape parameters.

The expected distance between two randomly chosen vertices or the average path length is one of the most important shape parameters in modeling complex networks as it indicates roughly how efficiently the information can be transmitted through the network. Following the same $\sqrt{n}$-vs- $\log n$ pattern, it is of order $\sqrt{n}$ in the simply-generated model, but $\log n$ in the increasing model. Another equally important parameter is the degree distribution of a random vertex: its limit distribution is a power law with exponential tails in the simply-generated model of the form $d^{-3 / 2} \rho_{k}^{d}$, in contrast to a power-law in the increasing model of the form 
$d^{-1-k /(k-1)}, d$ denoting the degree [28]. As regards the degree of the root-vertex ${ }^{1}$, its asymptotic distribution remains the same as that of any vertex in the simply-generated model, but in the increasing model, the root-degree distribution is different, with an asymptotic stable law (which is Rayleigh in the case $k=2$ ); see Theorem 3.

Our main concern in this paper is the connectivity-profile. Recall that the profile of an usual tree is the sequence of numbers, each enumerating the total number of nodes with the same distance to the root. For example, the tree - $<-<$ can be described by the profile $\{1,2,2,1,3\}$. Profiles represent one of the richest shape measures and they convey much information regarding particularly the silhouette. On random trees, they have been extensively studied recently; see $[10,19,20,27,32,36,44]$. Since $k$-trees have many cycles for $k \geq 2$, we call the profile of the shortest-path tree (see next section) the connectivity-profile as it measures to some extent the connectivity of the graph on the one hand and is well connected to the notion of profiles of trees on the other hand. Indeed this connectivity-profile corresponds to the profile of the "shortest-path tree" of a $k$-tree, as defined by Proskurowski [46], which is nothing more than the result of a Breadth First Search (BFS) on the graph. Moreover, in the domain of complex networks, this kind of BFS trees is an important object; for example, it describes the results of the traceroute measuring tool $[50,51]$ in the study of the topology of the Internet.

We will derive precise asymptotic approximations to the expected connectivity-profile of random increasing $k$-trees, the major tools used being based on the resolution of a system of differential equations of Cauchy-Euler type (see [13]). In particular, the expected number of nodes at distance $d$ from the root-vertex follows asymptotically a Gaussian distribution, in contrast to the Rayleigh limit distribution in the case of simply-generated $k$-trees. Also the limit distribution of the number of nodes with distance $d$ to the root-vertex will be derived when $d$ is bounded. Note that when $d=1$, the number of nodes at distance 1 to the root-vertex is nothing but the degree of the root-vertex. For other properties of random $k$-trees, see [28, 39, 43, 47].

This paper is organized as follows. We first present the definition and combinatorial specification of random increasing $k$-trees in Section 2, together with the enumerative generating functions, on which our analytic tools will be based. In Section 3, we present two asymptotic approximations to the expected connectivity-profile, one for $d=o(\log n)$ and the other for $d \rightarrow \infty$ and $d=O(\log n)$; and interesting consequences of our results are also given. A subsection is devoted to the proofs of the results. In Section 4 we give the limit distribution of the connectivity-profile in the range when $d=O(1)$. Finally the last section briefly provides some information on the methods of sampling $k$-trees that were used for the simulations.

\section{Random increasing $k$-trees and generating functions}

Since $k$-trees are graphs full of cycles and cliques, the key step in our analytic-combinatorial approach is to introduce a bijection between $k$-trees and a suitably defined class of trees for which generating functions can be derived. This approach was successfully applied to simplygenerated family of $k$-trees in [15], which leads to a system of algebraic equations. The bijection argument used there can be adapted mutatis mutandis here for increasing $k$-trees, which then yields a system of DEs through the bijection with a class of increasing trees [6].

\footnotetext{
${ }^{1}$ We call root-clique the clique composed of the $k$ vertices $(1, \ldots, k)$. The increasing nature of the $k$-trees guarantees that these vertices always form a clique. We call root-vertex the vertex with label 1.
} 

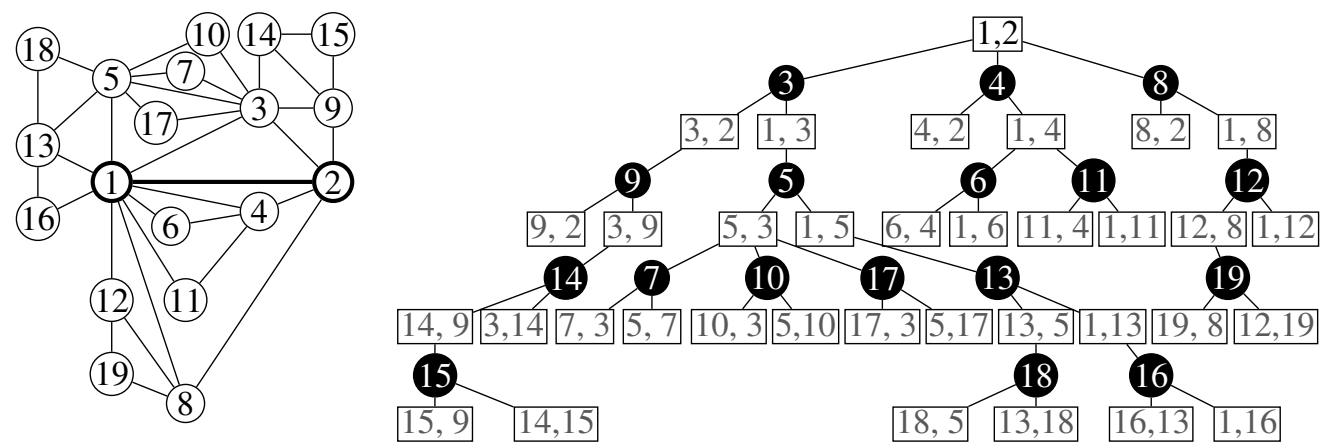

Figure 2: A 2-tree (left) and its corresponding increasing tree representation (right).

\subsection{Increasing $k$-trees and the bijection.}

Recall that a $k$-clique is a set of $k$ mutually adjacent vertices. A formal definition of increasing $k$-trees is as follows.

Definition 1. An increasing $k$-tree is defined recursively as follows. A $k$-clique in which each vertex gets a distinct label from $\{1, \ldots, k\}$ is an increasing $k$-tree of $k$ vertices. An increasing $k$-tree with $n>k$ vertices is constructed from an increasing $k$-tree with $n-1$ vertices by adding a vertex labeled $n$ and by connecting it by an edge to each of the $k$ vertices in an existing $k$-clique.

By random increasing $k$-trees, we assume that all existing $k$-cliques are equally likely each time a new vertex is being added. Given the fact that each time a vertex is added, $k$ new cliques appear in the random increasing $k$-tree, it is immediate that the number $T_{n}$ of increasing $k$-trees of $n+k$ nodes is given by $T_{n}=\prod_{0 \leq i<n}(i k+1)$.

Note that if we allow any permutation on all labels, we obtain the class of simply-generated $k$-trees where monotonicity of labels along paths fails in general.

Combinatorially, simply-generated $k$-trees are in bijection [15] with the family of trees specified by $\mathcal{K}_{s}=\mathcal{Z}^{k} \times \mathcal{T}_{s}$, where $\mathcal{T}_{s}=\operatorname{Set}\left(\mathcal{Z} \times \mathcal{T}_{s}^{k}\right)$. Given a rooted $k$-tree $G$ of $n$ vertices, we can transform $G$ into a tree $T$, with the root node labeled $\{1, \ldots, k\}$, by the following procedure.

1. First, associate a white node to each $k$-clique of $G$ and a black node to each $(k+1)$-clique of $G$.

2. Then add a link between each black node and all white nodes associated with the $k$ cliques it contains.

3. Each black node is labeled with the only vertex not appearing in one of the black nodes above it or in the root.

4. The last step is to order the $k$ vertices of the root and propagate this order to the $k$ sons of each black node.

This constructs a tree from a $k$-tree (see Figure 2); conversely, we can obtain the $k$-tree through a simple traversal of the tree. 
Such a bijection translates directly to increasing $k$-trees by restricting the class of corresponding trees to those respecting a monotonicity constraint on the labels, namely, on any path from the root to a leaf the labels are in increasing order. This yields the combinatorial specification of the class of increasing trees $\mathcal{T}=\operatorname{Set}\left(\mathcal{Z}^{\square} \times \mathcal{T}^{k}\right)$. An increasing $k$-tree is just a tree in $\mathcal{T}$ together with the sequence $\{1, \ldots, k\}$ corresponding to the labels of the root-clique. A tree in $\mathcal{K}=\mathcal{Z}^{\square} \times \cdots \times \mathcal{Z}^{\square} \times \mathcal{T}$ is thus completely determined by its $\mathcal{T}$ component, giving $\mathcal{K}_{n+k} \equiv \mathcal{T}_{n}$. In the rest of this paper we will thus focus on the class $\mathcal{T}$.

For example, Figure 2 shows a 2-tree with 19 vertices and its tree representation with 17 black nodes. The root-vertex is the vertex with label 1. It has 10 neighbors, including the vertex with label 2 which belongs to the root-clique; so it has degree 10, or equivalently there are 10 vertices at distance 1 to the root-vertex. All the other vertices are at distance 2 to the root-vertex, except for the vertex with label 15 , which is at distance 3 . The connectivity-profile of this 2 -tree is thus $\{1,10,7,1\}$.

\subsection{Generating functions.}

Following the bijection, we see that the complicated dependence structure of $k$-trees is now completely described by the class of increasing trees specified by $\mathcal{T}=\operatorname{Set}\left(\mathcal{Z}^{\square} \times \mathcal{T}^{k}\right)$. Let $T(z):=\sum_{n \geq 0} T_{n} z^{n} / n$ ! denote the exponential generating function of the number $T_{n}$ of increasing $k$-trees of $n+k$ vertices. Then, since the box construction $\mathcal{Z}^{\square} \times \mathcal{A}$ translates into the primitive $\int_{0}^{z} A(x) \mathrm{d} x$, the specification of $\mathcal{T}$ translates into the equation

$$
T(z)=\exp \left(\int_{0}^{z} T^{k}(x) \mathrm{d} x\right)
$$

or, equivalently, $T^{\prime}(z)=T^{k+1}(z)$ with $T(0)=1$, which is solved to be

$$
T(z)=(1-k z)^{-1 / k},
$$

we then check that $T_{n}=\prod_{0 \leq i<n}(i k+1)$.

Root degree. Marking by $u$ the neighbors of the root-vertex in $\mathcal{T}$, we notice that one amongst the $k$ subtrees is special since it does not contain any neighbor of the root-vertex, while the $k-1$ others have the same structure as the whole tree. We thus obtain

$$
T(z, u)=\exp \left(u \int_{0}^{z} T(x) T^{k-1}(x, u) \mathrm{d} x\right)
$$

where the coefficient $n !\left[u^{\ell} z^{n}\right] T(z, u)$ denotes the number of increasing $k$-trees of size $n+k$ with root degree equal to $k+\ell-1$ (counting the $k-1$ neighbors of the root-vertex coming from the root-clique). Taking derivative with respect to $z$ on both sides and then solving the equation, we get the closed-form expression

$$
T(z, u)=\left(1-u\left(1-(1-k z)^{1-1 / k}\right)\right)^{-1 /(k-1)} .
$$


Connectivity profile. The above bijection transforms $k$-trees into ordinary increasing trees; we are interested in the profile of the shortest-path trees of the $k$-trees, and study the corresponding parameter in trees (which is more complex than a profile). Also the results provide more insight on the structure of random $k$-trees. Roughly, we expect that all vertices in $k$-trees are close, one at most of logarithmic order away from the other. The fine results we derive provide in particular an upper bound for that.

Let $X_{n ; d, j}$ denote the number of nodes at distance $d$ from $j$ vertices of the root-clique in a random $k$-tree of $n+k$ vertices. Let $T_{d, j}(z, u)=\sum_{n \geq 0} T_{n} \mathbb{E}\left(u^{X_{n ; d, j}}\right) z^{n} / n$ ! denote the corresponding bivariate generating function.

Theorem 1. The generating functions $T_{d, j}$ 's satisfy the DEs

$$
\frac{\partial}{\partial z} T_{d, j}(z, u)=u^{\delta_{d, 1}} T_{d, j-1}^{j}(z, u) T_{d, j}^{k-j+1}(z, u),
$$

with the initial conditions $T_{d, j}(0, u)=1$ for $1 \leq j \leq k$, where $\delta_{a, b}$ denotes the Kronecker function, $T_{0, k}(z, u)=T(z)$ and $T_{d, 0}(z, u)=T_{d-1, k}(z, u)$.

Proof. The theorem follows from the equation

$$
T_{d, j}(z, u)=\exp \left(u^{\delta_{d, 1}} \int_{0}^{z} T_{d, j-1}^{j}(x, u) T_{d, j}^{k-j}(x, u) \mathrm{d} x\right)
$$

with $T_{d, j}(z, 1)=T(z)$.

Notation. For operational convenience, we normalize all $z$ by $z / k$ and write

$$
\tilde{T}(z):=T(z / k)=(1-z)^{-1 / k} .
$$

Similarly, we define $\tilde{T}_{d, j}(z, u):=T_{d, j}(z / k, u)$ and have, by (2),

$$
\frac{\partial}{\partial z} \tilde{T}_{d, j}(z, u)=\frac{u^{\delta_{d, 1}}}{k} \tilde{T}_{d, j-1}^{j}(z, u) \tilde{T}_{d, j}^{k-j+1}(z, u),
$$

with $\tilde{T}_{d, j}(z, 1)=\tilde{T}(z), \tilde{T}_{0, k}(z, u)=\tilde{T}(z)$ and $\tilde{T}_{d, 0}(z, u)=\tilde{T}_{d-1, k}(z, u)$.

\section{Expected connectivity-profile}

In this section we state our major results for the expected value of the connectivity-profile $\left(X_{n ; d, j}\right)$, when $d=O(\log n)$. As interesting consequences, we also derive $(i)$ an explicit expression for the expected degree of the root-vertex, (ii) an asymptotic Gaussian approximation to the expected value of $\left(X_{n ; d, j}\right)$ when $d$ is close to $(\log n) /\left(k H_{k}\right)$, a region where most of the vertices are concentrated, where $H_{k}:=\sum_{1 \leq \ell \leq k} 1 / \ell$ denotes the harmonic numbers, (iii) an upper bounds for the height and (iv) a lower bound for the width of the tree. The proofs of these corollaries are given in the end of this section. 


\subsection{The expected value}

We consider the expected connectivity-profile, $\mathbb{E}\left(X_{n ; d, j}\right)$, which corresponds to the expected profile of the shortest-path trees of increasing $k$-trees. Observe first that

$$
\mathbb{E}\left(X_{n ; d, j}\right)=\frac{k^{n}\left[z^{n}\right] \tilde{M}_{d, j}(z)}{T_{n}}, \quad \text { where } \quad \tilde{M}_{d, j}(z):=\left.\frac{\partial \tilde{T}_{d, j}(z, u)}{\partial u}\right|_{u=1}
$$

It follows from (3) that

$$
\tilde{M}_{d, j}^{\prime}(z)=\frac{(k-j+1) \tilde{M}_{d, j}(z)+j \tilde{M}_{d, j-1}(z)}{k(1-z)}+\frac{\delta_{d, 1} \tilde{T}(z)}{k(1-z)} .
$$

This is a DE of standard Cauchy-Euler type whose solution is given by (see [13])

$$
\tilde{M}_{d, j}(z)=\frac{(1-z)^{-(k-j+1) / k}}{k} \int_{0}^{z}(1-x)^{-(j-1) / k}\left(j \tilde{M}_{d, j-1}(x)+\delta_{d, 1} \tilde{T}(x)\right) \mathrm{d} x,
$$

since $\tilde{M}_{d, j}(0)=0$. Then, starting from $\tilde{M}_{0, k}=0$, we get

$$
\tilde{M}_{1,1}(z)=\frac{1}{k-1}\left(\frac{1}{1-z}-\frac{1}{(1-z)^{1 / k}}\right) .
$$

And by induction, we get

$$
\tilde{M}_{d, j}(z) \sim \frac{j}{(k-1)(d-1) !} \cdot \frac{1}{1-z} \log ^{d-1} \frac{1}{1-z},
$$

for $1 \leq j \leq k, d \geq 1$ and $z \sim 1$. One can then prove, by singularity analysis (see [25, 24]), that

$$
\mathbb{E}\left(X_{n ; d, j}\right) \sim \Gamma(1 / k) \frac{j}{k-1} \cdot \frac{(\log n)^{d-1}}{(d-1) !} n^{1-1 / k},
$$

for large $n$ and fixed $d, k$ and $1 \leq j \leq k$.

In Theorem 2 below, we show that the same asymptotic estimate holds in the larger range $d=o(\log n)$, and also derive an estimate for $d=O(\log n)$. Let us first introduce the notation $\lambda_{1}(w), \ldots, \lambda_{k}(w)$ for the zeros of the equation $P_{k}(\theta)=\frac{k !}{k^{k}} w-\prod_{\ell=1}^{k}\left(\theta-\frac{\ell}{k}\right)$, with the $\lambda_{i}$ arranged in decreasing order of their real parts: $\Re\left(\lambda_{1}(w)\right) \geq \cdots \geq \Re\left(\lambda_{k}(w)\right)$.

Theorem 2. The expected connectivity-profile $\mathbb{E}\left(X_{n ; d, j}\right)$ satisfies for $1 \leq d=o(\log n)$

$$
\mathbb{E}\left(X_{n ; d, j}\right) \sim \Gamma(1 / k) \frac{j}{k-1} \cdot \frac{(\log n)^{d-1}}{(d-1) !} n^{1-1 / k},
$$

uniformly in $d$, and for $d \rightarrow \infty, d=O(\log n)$,

$$
\mathbb{E}\left(X_{n ; d, j}\right) \sim \frac{\Gamma(1 / k) h_{j, 1}(\rho) \rho^{-d} n^{\lambda_{1}(\rho)-1 / k}}{\Gamma\left(\lambda_{1}(\rho)\right) \sqrt{2 \pi\left(\rho \lambda_{1}^{\prime}(\rho)+\rho^{2} \lambda_{1}^{\prime \prime}(\rho)\right) \log n}}
$$

where $\rho=\rho_{n, d}>0$ solves the equation $\rho \lambda_{1}^{\prime}(\rho)=d / \log n$, and $h_{j, 1}$ is defined by

$$
h_{j, 1}(w)=\frac{j ! w(w-1)}{\left(k \lambda_{1}(w)-1\right) \prod_{k-j+1 \leq s \leq k+1}\left(k \lambda_{1}(w)-s\right)} \cdot \frac{1}{\sum_{1 \leq s \leq k} \frac{1}{k \lambda_{1}(w)-s}},
$$

for $1 \leq j \leq k$. 
This theorem cannot be proved by the above inductive argument and our method of proof, which is depicted in Figure 3, consists of the following steps. First, we consider the bivariate series $\mathscr{M}_{j}(z, w):=\sum_{d \geq 1} \tilde{M}_{d, j}(z) w^{d}$, which satisfies the linear system

$$
\left((1-z) \frac{\mathrm{d}}{\mathrm{d} z}-\frac{k-j+1}{k}\right) \mathscr{M}_{j}=\frac{j}{k} \mathscr{M}_{j-1}+\frac{w \tilde{T}}{k},
$$

for $1 \leq j \leq k$. Second, this system is solved and has the solutions

$$
\mathscr{M}_{j}(z, w)=\sum_{1 \leq j \leq k} h_{j, m}(w)(1-z)^{-\lambda_{m}(w)}-\tilde{T}(z) \frac{w-(w-1) \delta_{k, j}}{k},
$$

where the $h_{j, m}$ have the same expression as $h_{j, 1}$ but with all $\lambda_{1}(w)$ in (8) replaced by $\lambda_{m}(w)$. While the form of the solution is well anticipated, the hard part is the calculations of the coefficient-functions $h_{j, m}$. Third, by singularity analysis and a delicate study of the zeros, we then conclude, by saddle-point method, the estimates given in the theorem.

The complete proof of theorem 2 is given in Section 3.3.

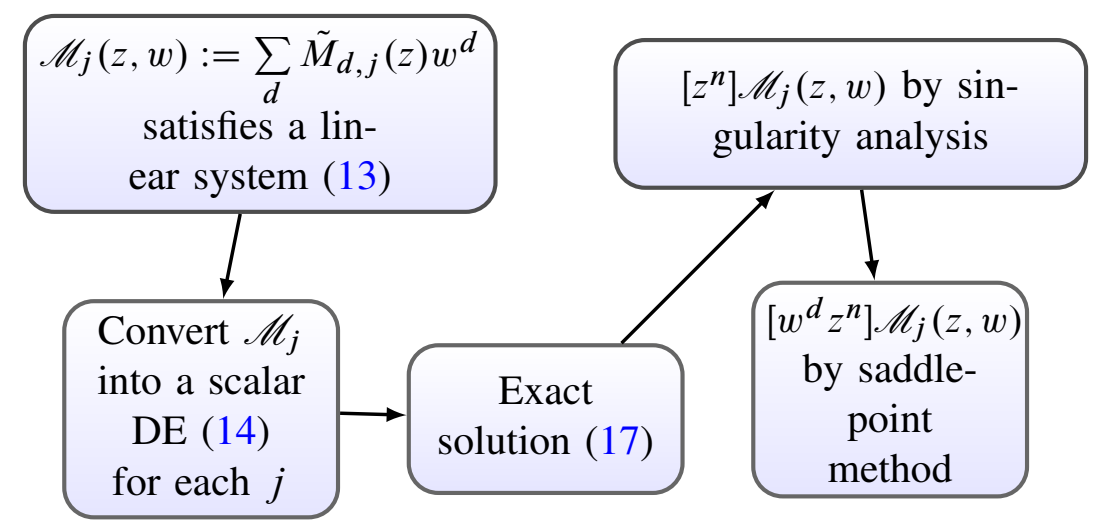

Figure 3: Major steps used in the proof of Theorem 2.

\subsection{Corollaries}

The asymptotic approximations to the mean profile entail interesting properties concerning the structure of random increasing $k$-trees, notably the distances to the root-vertex.

Expected root-degree. Setting $d=1$ in expression (6), we obtain an estimate for the expected degree of the root-vertex.

Corollary 1. The expected degree of the root-vertex $\mathbb{E}\left(X_{n, 1, j}\right)$ satisfies

$$
\mathbb{E}\left(X_{n, 1, j}\right) \sim \Gamma(1 / k) \frac{j}{k-1} n^{1-1 / k} \quad(1 \leq j \leq k) .
$$


Gaussian approximation. As a consequence of expression (7), the expected connectivityprofile can be approximated by a Gaussian law when $d$ is around $\log n$. More precisely, let $H_{k}:=\sum_{1 \leq \ell \leq k} 1 / \ell$ denote the harmonic numbers and $H_{k}^{(2)}:=\sum_{1 \leq \ell \leq k} 1 / \ell^{2}$.

Corollary 2. The expected number of nodes at distance $d=\left\lfloor\frac{1}{k H_{k}} \log n+x \sigma \sqrt{\log n}\right\rfloor$ from the root-vertex, where $\sigma=\sqrt{H_{k}^{(2)} /\left(k H_{k}^{3}\right)}$, satisfies, uniformly for $x=o\left((\log n)^{1 / 6}\right)$,

$$
\mathbb{E}\left(X_{n ; d, j}\right) \sim \frac{n e^{-x^{2} / 2}}{\sqrt{2 \pi \sigma^{2} \log n}} .
$$

The proof is given in Section 3.4.

This Gaussian approximation justifies the last item corresponding to increasing trees in Table 1; see Figure 4 for a graphical display.

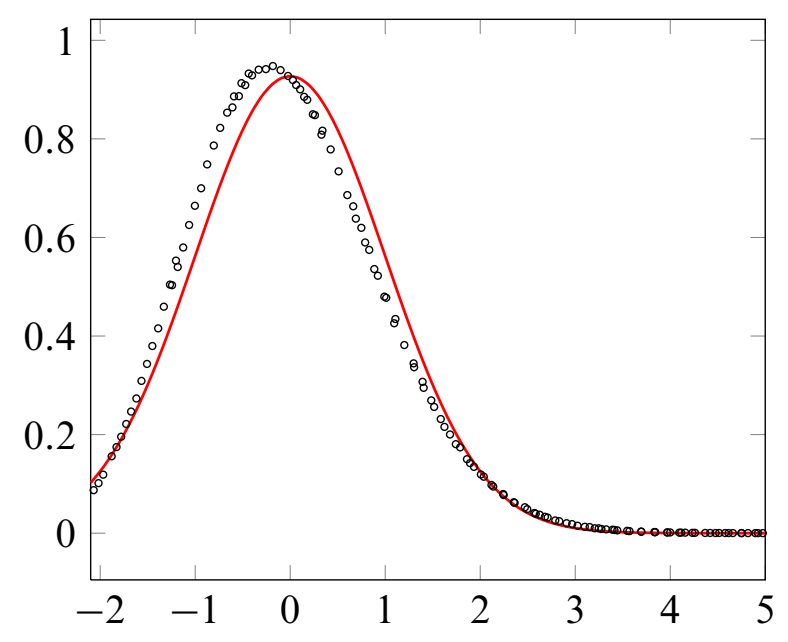

Figure 4: A graphical rendering of Corollary 2. The simulation result of $\mathbb{E}\left(X_{n ; d, j}\right) /(n / \sqrt{\log n})$ plotted against $x:=\left(d-\frac{1}{2}-\frac{\log n}{k H_{k}}\right) /(\sigma \sqrt{\log n})$. The red curve represents the corresponding Gaussian density curve $e^{-x^{2} / 2} / \sqrt{2 \pi \sigma^{2}}$. The slight disagreement of the two curves is mainly due to the integer part (d can assume only integers).

Expected height and width. The estimate we derived for the expected profile provides an upper bound for the height and a lower bound for the width (of the shortest-path tree) of a random increasing $k$-tree.

Corollary 3. Let $\mathscr{H}_{n}$ denote the height of a random increasing $k$-tree of $n+k$ vertices. Then

$$
\mathbb{E}\left(\mathscr{H}_{n}\right) \leq \alpha_{+} \log n-\frac{\alpha_{+}}{2\left(\lambda_{1}\left(\alpha_{+}\right)-\frac{1}{k}\right)} \log \log n+O(1),
$$


where $\alpha_{+}>0$ is the solution of the system of equations

$$
\left\{\begin{array}{l}
\frac{1}{\alpha_{+}}=\sum_{1 \leq \ell \leq k} \frac{1}{v-\frac{\ell}{k}} \\
v-\frac{1}{k}-\alpha_{+} \sum_{1 \leq \ell \leq k} \log \left(\frac{k}{\ell} v-1\right)=0 .
\end{array}\right.
$$

See Section 3.4 for the proof.

Table 2 gives the numerical values of $\alpha_{+}$for small values of $k$. For large $k$, one can show

\begin{tabular}{|c||c|c|c|c|c|}
\hline$k$ & 2 & 3 & 4 & 5 & 6 \\
\hline$\alpha_{+}$ & 1.085480 & 0.656285 & 0.465190 & 0.358501 & 0.290847 \\
\hline \hline$k$ & 7 & 8 & 9 & 10 & 20 \\
\hline$\alpha_{+}$ & 0.244288 & 0.210365 & 0.184587 & 0.164356 & 0.077875 \\
\hline
\end{tabular}

Table 2: Approximate numerical values of $\alpha_{+}$.

that $\alpha_{+} \sim 1 /(k \log 2)$ and $\lambda_{1}\left(\alpha_{+}\right) \sim 2$.

Corollary 3 justifies that the mean distance of random $k$-trees are of logarithmic order in size, as stated in Table 1.

In addition to height, we also have a lower bound for the width, which is the maximum number of nodes that appears on a single distance layer $\mathscr{W}_{n}:=\max _{d} X_{n ; d, j}$.

Corollary 4. The width of a random increasing $k$-tree of $n+k$ vertices is bounded from below by

$$
\mathbb{E}\left(\mathscr{W}_{n}\right)=\mathbb{E}\left(\max _{d} X_{n, d}\right) \geq \max _{d} \mathbb{E}\left(X_{n, d}\right) \sim \frac{n}{\sqrt{2 \pi \sigma^{2} \log n}} .
$$

We may conclude briefly from these results that in the shortest-path increasing trees of random increasing $k$-trees, almost all nodes are located at the levels with $d=\frac{1}{k H_{k}} \log n+$ $O(\sqrt{\log n})$, each with $\approx n / \sqrt{\log n}$ nodes, similar to most search trees of logarithmic height (see [27]). Our fine estimates also provide additional clarification of the small-world nature of random $k$-trees, notably $k=3$ for which different claims on the average path-length over a deterministic 3-tree model (Apollonian networks) were proposed in [1] and in [53]. Strangely, while the models are different, our asymptotic result $\frac{2}{11} \log n$ is consistent with that derived in [53]; see also $[36,26]$ for related results. As the sequence $1 /\left(k H_{k}\right)$ decreases moderately for increasing $k$ and $\log n$ increases only smoothly, wrong conclusions may be drawn in practice based solely on numerical evidence.

\subsection{Proof of theorem 2}

We prove in this section Theorem 2 on the asymptotic approximations to the expected connectivityprofile. A diagrammatic sketch of the major steps of our proof is already given in Figure 3. 


\subsubsection{Bivariate generating function and the linear system}

Our approach to the asymptotics of $\mathbb{E}\left(X_{n ; d, j}\right)$ begins with the bivariate generating functions that gather information on the number of nodes at distance $d$ from $j$ vertices of the root-clique for every value of $d$

$$
\mathscr{M}_{j}(z, w):=\sum_{d \geq 1} \tilde{M}_{d, j}(z) w^{d}
$$

Lemma 1. The bivariate generating functions $\mathscr{M}_{j}$ satisfy the linear system

$$
\left\{\begin{aligned}
(\theta-1) \mathscr{M}_{1} & =\frac{w}{k} \mathscr{M}_{k}+\frac{w \tilde{T}}{k} \\
\left(\theta-\frac{k-1}{k}\right) \mathscr{M}_{2} & =\frac{2}{k} \mathscr{M}_{1}+\frac{w \tilde{T}}{k} \\
\vdots & \vdots \\
\left(\theta-\frac{1}{k}\right) \mathscr{M}_{k} & =\mathscr{M}_{k-1}+\frac{w \tilde{T}}{k} .
\end{aligned}\right.
$$

with the operator $\theta:=(1-z) \mathbb{D}_{z}$.

Proof. By (4), we obtain for $j=1, \ldots, k$

$$
\frac{\partial}{\partial z} \mathscr{M}_{j}(z, w)=\frac{k-j+1}{k(1-z)} \mathscr{M}_{j}(z, w)+\frac{j}{k(1-z)} \mathscr{M}_{j-1}+\frac{w \tilde{T}(z)}{k(1-z)} .
$$

Moreover

$$
\mathscr{M}_{0}(z, w)=\sum_{d \geq 1} \tilde{M}_{d, 0}(z) w^{d}=\sum_{d \geq 1} \tilde{M}_{d-1, k}(z) w^{d}=w \mathscr{M}_{k}(z, w)
$$

Thus (13) follows.

The easy special case is when $k=1$, which has the exact solution

$$
\begin{aligned}
\mathscr{M}_{1}(z, w) & =w(1-z)^{-w-1} \int_{0}^{z}(1-t)^{w-1} \mathrm{~d} t \\
& =(1-z)^{-w-1}-(1-z)^{-1},
\end{aligned}
$$

leading to Stirling numbers of the first kind; see [24].

Remark. The system (13) can easily be cast into the matrix form

$$
\theta \mathbf{M}-\mathbf{A} \mathbf{M}=\mathbf{g},
$$

where

$$
\mathbf{M}(z):=\left(\begin{array}{c}
\mathscr{M}_{1} \\
\mathscr{M}_{2} \\
\vdots \\
\mathscr{M}_{k}
\end{array}\right)(z, w), \quad \mathbf{g}(z):=\frac{w \tilde{T}}{k}\left(\begin{array}{c}
1 \\
1 \\
\vdots \\
1
\end{array}\right)(z)
$$


and

$$
\mathbf{A}:=\frac{1}{k}\left(\begin{array}{ccccc}
k & 0 & \cdots & \cdots & w \\
2 & k-1 & 0 & \cdots & 0 \\
0 & 3 & k-2 & \cdots & 0 \\
\vdots & \vdots & \ddots & \ddots & \vdots \\
0 & 0 & \cdots & k & 1
\end{array}\right) .
$$

Let $\boldsymbol{\Omega}(z):=(1-z)^{\mathbf{A}}=\exp (\mathbf{A} \log (1-z))$ be the fundamental matrix function. Then

$$
\boldsymbol{\Omega}(\theta \mathbf{M}-\mathbf{A M})=\theta(\boldsymbol{\Omega} \mathbf{M})=\mathbf{\Omega} \mathbf{g},
$$

so that the solution is given by $(\mathbf{M}(0)=\mathbf{0})$

$$
\mathbf{M}(z)=\boldsymbol{\Omega}(z)^{-1} \int_{0}^{z} \frac{1}{1-x} \boldsymbol{\Omega}(x) \mathbf{g}(x) \mathrm{d} x .
$$

With this form of solution, one can derive explicit forms for $\mathbf{M}(z)$ for small values of $k$, but the calculations soon become intractable for larger $k$ as it involves the matrix exponential $\boldsymbol{\Omega}$ and its inverse.

\subsubsection{Converting the linear system (13) into scalar DEs}

Instead of working directly on the matrix form, we convert the linear system into $k$ scalar DEs of Cauchy-Euler type, with the same differential operator for all equations.

Proposition 1. Let $c_{k}:=k ! / k^{k}$. The bivariate generating functions $\mathscr{M}_{j}$ satisfy the following $D E s$

$$
\begin{aligned}
& {\left[\prod_{1 \leq \ell \leq k}\left(\theta-\frac{\ell}{k}\right)\right] \mathscr{M}_{j}=c_{k} w \mathscr{M}_{j}+\frac{c_{k}}{k} w^{2} \tilde{T} \quad(1 \leq j<k),} \\
& {\left[\prod_{1 \leq \ell \leq k}\left(\theta-\frac{\ell}{k}\right)\right] \mathscr{M}_{k}=c_{k} w \mathscr{M}_{k}+\frac{c_{k}}{k} w \tilde{T},}
\end{aligned}
$$

where the initial conditions differ in each equation.

A particular solution is easily seen to be $-w(1-z)^{-1 / k} / k$ for $\mathscr{M}_{j}$ for $1 \leq j<k$, and $-(1-z)^{-1 / k} / k$ for $\mathscr{M}_{k}$.

Our approach to converting the system (13) is to consider $\left[\prod_{1 \leq \ell \leq k}\left(\theta-\frac{\ell}{k}\right)\right] \mathscr{M}_{j}$, and then simplify this by applying successively the equations in (13) one after another, so as to obtain a scalar DE for each $\mathscr{M}_{j}$.

Proof. We start with the proof for $\mathscr{M}_{1}$, the others then following by induction.

$$
\begin{aligned}
{\left[\prod_{1 \leq \ell \leq k}\left(\theta-\frac{\ell}{k}\right)\right] \mathscr{M}_{1} } & =\left[\prod_{1 \leq \ell<k}\left(\theta-\frac{\ell}{k}\right)\right](\theta-1) \mathscr{M}_{1} \\
& =\left[\prod_{1 \leq \ell<k}\left(\theta-\frac{\ell}{k}\right)\right]\left(\frac{w}{k} \mathscr{M}_{k}+\frac{w \tilde{T}}{k}\right) \\
& =\frac{w}{k}\left[\prod_{1 \leq \ell<k}\left(\theta-\frac{\ell}{k}\right)\right] \mathscr{M}_{k},
\end{aligned}
$$


since $\left(\tilde{T}(z)=(1-z)^{-1 / k}\right)$

$$
\left[\prod_{1 \leq \ell<k}\left(\theta-\frac{\ell}{k}\right)\right] \tilde{T}=\tilde{T} \prod_{1 \leq \ell<k}\left(\frac{1}{k}-\frac{\ell}{k}\right)=0 .
$$

Iterating this procedure of linear operators, we finally get

$$
\begin{aligned}
& {\left[\prod_{1 \leq \ell \leq k}\left(\theta-\frac{\ell}{k}\right)\right] \mathscr{M}_{1}} \\
& \quad=c_{k} w \mathscr{M}_{1}+\frac{c_{k}}{2} w^{2} \tilde{T}+w^{2} \tilde{T} \sum_{2 \leq s<k} \frac{k !}{k^{s}(k-s+2) !} \prod_{s \leq \ell<k}\left(\frac{1}{k}-\frac{\ell}{k}\right),
\end{aligned}
$$

and the last two terms on the right-hand side simplify to $(k-1) ! w^{2} \tilde{T} / k^{k}$. Although this approach also applies to other $\mathscr{M}_{j}$, it is simpler to use the following argument once we proved (14) for $j=1$. We assume first the form

$$
\left[\prod_{1 \leq \ell \leq k}\left(\theta-\frac{\ell}{k}\right)\right] \mathscr{M}_{j}=c_{k} w \mathscr{M}_{j}+C_{k, j} w^{2} \tilde{T},
$$

for $1 \leq j<k$, which is already proved for $j=1$. Consider now $2 \leq j<k$.

$$
\begin{aligned}
{\left[\prod_{1 \leq \ell \leq k}\left(\theta-\frac{\ell}{k}\right)\right] \mathscr{M}_{j} } & =\left[\prod_{\ell \neq k-j+1}\left(\theta-\frac{\ell}{k}\right)\right]\left(\theta-\frac{k-j+1}{k}\right) \mathscr{M}_{j} \\
& =\frac{j}{k}\left[\prod_{\ell \neq k-j+1}\left(\theta-\frac{\ell}{k}\right)\right] \mathscr{M}_{j-1} .
\end{aligned}
$$

Multiplying the missing factor $\theta-(k-j+1) / k$, we obtain

$$
\frac{j}{k}\left[\prod_{1 \leq \ell \leq k}\left(\theta-\frac{\ell}{k}\right)\right] \mathscr{M}_{j-1}=\frac{j}{k}\left(c_{k} w \mathscr{M}_{j-1}+C_{k, j-1} w^{2} \tilde{T}\right) ;
$$

which, on the other hand, also equals, by (15),

$$
\begin{aligned}
(\theta- & \left.\frac{k-j+1}{k}\right)\left(c_{k} w \mathscr{M}_{j}+C_{k, j} w^{2} \tilde{T}\right) \\
& =c_{k} w\left(\frac{j}{k} \mathscr{M}_{j-1}+\frac{w \tilde{T}}{k}\right)-\frac{k-j}{k} C_{k, j} w^{2} \tilde{T} .
\end{aligned}
$$

Thus equating these two last displays, we get the recurrence for $C_{k, j}$

$$
C_{k, j}=\frac{1}{k-j}\left(\frac{k !}{k^{k}}-j C_{k, j-1}\right) \quad(2 \leq j<k),
$$

with the initial condition $C_{k, 1}=(k-1) ! / k^{k}$. It is then easily verified that $C_{k, j} \equiv(k-$ $1) ! / k^{k}=c_{k} / k$ for $2 \leq j<k$. 


\subsubsection{Solution to the Cauchy-Euler DEs (14)}

To solve the Cauchy-Euler DEs (14), we first examine the zeros of the indicial equation

$$
P_{k}(\theta):=\prod_{1 \leq \ell \leq k}\left(\theta-\frac{\ell}{k}\right)-c_{k} w=\prod_{1 \leq j \leq k}\left(\theta-\lambda_{j}(w)\right),
$$

where $\Re\left(\lambda_{1}(w)\right) \geq \Re\left(\lambda_{2}(w)\right) \geq \cdots \geq \Re\left(\lambda_{k}(w)\right)$. Then for $w \neq 0$, it can be proved that all zeros are simple.

Proposition 2. The exact solution of (14) is given by

$$
\mathscr{M}_{j}(z, w)=\sum_{1 \leq m \leq k} h_{j, m}(w)(1-z)^{-\lambda_{m}(w)}-\frac{w-(w-1) \delta_{k, j}}{k} \tilde{T}(z),
$$

where the $h_{j, m}$ 's are given in (23) and (24).

In the special case when $k=2$, the two solutions of $P_{2}(\theta)=0$ are given by

$$
\lambda_{1}(w)=\frac{3+\sqrt{1+8 w}}{4} \text { and } \quad \lambda_{2}(w)=\frac{3-\sqrt{1+8 w}}{4}
$$

and the exact solution to (14) with initial conditions zero can be easily found to be

$$
\begin{aligned}
& \mathscr{M}_{1}(z, w)=\frac{w(3+\sqrt{1+8 w})}{4 \sqrt{1+8 w}}(1-z)^{-\lambda_{1}(w)}-\frac{w(3-\sqrt{1+8 w})}{4 \sqrt{1+8 w}}(1-z)^{-\lambda_{2}(w)}-\frac{w}{2}(1-z)^{-1 / 2}, \\
& \mathscr{M}_{2}(z, w)=\frac{4 w-1+\sqrt{1+8 w}}{4 \sqrt{1+8 w}}(1-z)^{-\lambda_{1}(w)}-\frac{4 w-1-\sqrt{1+8 w}}{4 \sqrt{1+8 w}}(1-z)^{-\lambda_{2}(w)}-\frac{1}{2}(1-z)^{-1 / 2},
\end{aligned}
$$

since $\tilde{T}(z)=(1-z)^{-1 / k}$; see [11, Corollary 4, p. 323]. But the expressions become more involved for higher values of $k$.

Proof of Proposition 2. We now prove Proposition 2 by a linear-operator approach (to obtain $h_{k, m}$ ) and then by induction (for other $h_{j, m}$ 's).

A linear-operator approach to deriving the coefficients $h_{j, m}$. To derive explicit expressions for $h_{j, m}$, we apply a linear-operator approach, often used for Cauchy-Euler DEs; see for example $[29,30,12,13]$.

The idea is roughly as follows. We focus on $\mathscr{M}_{k}$; the other cases can then be derived by (13). By (16), we define

$$
\mathscr{M}_{k}^{[1]}:=\left(\theta-\lambda_{2}(w)\right) \cdots\left(\theta-\lambda_{k}(w)\right) \mathscr{M}_{k},
$$

so that the DE (14) can be written as the first-order DE

$$
\left(\theta-\lambda_{1}(w)\right) \mathscr{M}_{k}^{[1]}=\frac{c_{k}}{k} w T
$$


which has the solution

$$
\begin{aligned}
\mathscr{M}_{k}^{[1]}(z) & =\mathscr{M}_{k}^{[1]}(0)(1-z)^{-\lambda_{1}(w)}+\frac{c_{k}}{k} w(1-z)^{-\lambda_{1}(w)} \int_{0}^{z}(1-x)^{\lambda_{1}(w)-1-1 / k} \mathrm{~d} x \\
& =\left(\mathscr{M}_{k}^{[1]}(0)+\frac{c_{k} w}{k \lambda_{1}(w)-1}\right)(1-z)^{-\lambda_{1}(w)}-\frac{c_{k}}{k \lambda_{1}(w)-1}(1-z)^{-1 / k} .
\end{aligned}
$$

All coefficients are explicit except $\mathscr{M}_{k}^{[1]}(0)$. To derive a more explicit expression for $\mathscr{M}_{k}^{[1]}(0)$, we start with the identity

$$
\frac{\prod_{1 \leq \ell \leq k}\left(\theta-\frac{\ell}{k}\right)-c_{k} w}{\theta-\lambda}=\sum_{0 \leq s<k}\left[\prod_{s+2 \leq \ell \leq k}\left(\lambda-\frac{\ell}{k}\right)\right]\left[\prod_{1 \leq \ell \leq s}\left(\theta-\frac{\ell}{k}\right)\right],
$$

whenever $\lambda \in\left\{\lambda_{1}(w), \ldots, \lambda_{k}(w)\right\}$, namely $P_{k}(\lambda)=0$. To prove this, we observe that

$$
\begin{aligned}
\frac{\prod_{1 \leq \ell \leq k}\left(\theta-\frac{\ell}{k}\right)}{\theta-\lambda} & =\frac{\left[\prod_{1 \leq \ell<k}\left(\theta-\frac{\ell}{k}\right)\right]\left(\theta-\lambda+\lambda-\frac{k}{k}\right)}{\theta-\lambda} \\
& =\prod_{1 \leq \ell<k}\left(\theta-\frac{\ell}{k}\right)+(\lambda-1) \frac{\prod_{1 \leq \ell<k}\left(\theta-\frac{\ell}{k}\right)}{\theta-\lambda} \\
& =\cdots \\
& =\sum_{0 \leq s<k}\left[\prod_{s+2 \leq \ell \leq k}\left(\lambda-\frac{\ell}{k}\right)\right]\left[\prod_{1 \leq \ell \leq s}\left(\theta-\frac{\ell}{k}\right)\right]+\frac{\prod_{1 \leq \ell \leq k}\left(\lambda-\frac{\ell}{k}\right)}{\theta-\lambda} .
\end{aligned}
$$

Now adding $-c_{k} w /(\theta-\lambda)$ to both sides has the effect of canceling the last term on the right-and side since $P_{k}(\lambda)=0$.

From (19), we have

$$
\mathscr{M}_{k}^{[1]}(z)=\sum_{0 \leq s<k}\left[\prod_{s+2 \leq \ell \leq k}\left(\lambda_{1}(w)-\frac{\ell}{k}\right)\right]\left[\prod_{1 \leq \ell \leq s}\left(\theta-\frac{\ell}{k}\right)\right] \mathscr{M}_{k}(z) .
$$

This expression provides an effective means to compute the value $\mathscr{M}_{k}^{[1]}(0)$. To that purpose, we observe first, by induction, that

$$
\left.\prod_{1 \leq \ell \leq s}\left(\theta-\frac{\ell}{k}\right) \mathscr{M}_{k}(z)\right|_{z=0}=\frac{(k-1) \cdots(k-s+1)}{k^{s}} w,
$$

for $1 \leq s \leq k$. This, together with $\mathscr{M}_{k}(0)=0$, gives

$$
\begin{aligned}
\mathscr{M}_{k}^{[1]}(0) & =w \sum_{1 \leq s<k}\left[\prod_{s+2 \leq \ell \leq k}\left(\lambda_{1}(w)-\frac{\ell}{k}\right)\right] \frac{k !}{(k-s) ! k^{s+1}} \\
& =c_{k} w \sum_{1 \leq s<k} \frac{\Gamma\left(k \lambda_{1}(w)-s-1\right)}{\Gamma\left(k \lambda_{1}(w)-k\right) \Gamma(k-s+1)} \\
& =\frac{c_{k} w}{k \lambda_{1}(w)-k-1}\left(\frac{k w}{k \lambda_{1}(w)-1}-1\right) \\
& =\frac{c_{k} w\left(k w-k \lambda_{1}(w)+1\right)}{\left(k \lambda_{1}(w)-k-1\right)\left(k \lambda_{1}(w)-1\right)} .
\end{aligned}
$$


Substituting this into (18) and then iterating the linear operators, we deduce that (see, for example, [13])

$$
\begin{aligned}
h_{k, 1}(w) \prod_{2 \leq \ell \leq k}\left(\lambda_{1}(w)-\lambda_{\ell}(w)\right) & =\frac{c_{k} w\left(k w-k \lambda_{1}(w)+1\right)}{\left(k \lambda_{1}(w)-k-1\right)\left(k \lambda_{1}(w)-1\right)}+\frac{c_{k} w}{k \lambda_{1}(w)-1} \\
& =\frac{c_{k} k w(w-1)}{\left(k \lambda_{1}(w)-k-1\right)\left(k \lambda_{1}(w)-1\right)} .
\end{aligned}
$$

The same procedure applies to other $h_{k, m}(w)$ 's, and we obtain

$$
h_{k, m}(w)=\frac{c_{k} k w(w-1)}{\left(k \lambda_{m}(w)-k-1\right)\left(k \lambda_{m}(w)-1\right) \prod_{\ell \neq m}\left(\lambda_{m}(w)-\lambda_{\ell}(w)\right)} \quad(1 \leq m \leq k) .
$$

Now, again by (19),

$$
\begin{aligned}
\prod_{\ell \neq m}\left(\lambda_{m}(w)-\lambda_{\ell}(w)\right) & =\lim _{\theta \rightarrow \lambda_{m}(w)} \frac{\prod_{1 \leq \ell \leq k}\left(\theta-\lambda_{\ell}(w)\right)}{\theta-\lambda_{m}(w)} \\
& =\sum_{0 \leq s<k} \prod_{\ell \neq s+1}\left(\lambda_{m}(w)-\frac{\ell}{k}\right) \\
& =k c_{k} w \sum_{1 \leq s \leq k} \frac{1}{k \lambda_{m}(w)-s} .
\end{aligned}
$$

It follows that

$$
h_{k, m}(w)=\frac{w-1}{\left(k \lambda_{m}(w)-k-1\right)\left(k \lambda_{m}(w)-1\right) \sum_{1 \leq s \leq k} \frac{1}{k \lambda_{m}(w)-s}} \quad(1 \leq m \leq k),
$$

provided that $k \lambda_{m}(w) \not s, s=2, \ldots, m$, which occurs when $w \sim 0$. In this case, we use the more general but less explicit expression

$$
h_{k, m}(w)=\frac{(w-1) \prod_{2 \leq s \leq k}\left(k \lambda_{m}(w)-s\right)}{k ! w\left(k \lambda_{m}(w)-k-1\right) \sum_{1 \leq s \leq k} \frac{1}{k \lambda_{m}(w)-s}} \quad(1 \leq m \leq k),
$$

which also follows from (20). Properties of the $\lambda_{m}$ 's will be discussed below.

The recurrence relation for $h_{j, m}(w)$. From the linear system (13), we see that, by (17),

$$
\begin{aligned}
(\theta- & \left.\frac{k-j+1}{k}\right) \mathscr{M}_{j}(z) \\
& =\sum_{1 \leq m \leq k} h_{j, m}(w)\left(\lambda_{m}(w)-\frac{k-j+1}{k}\right)(1-z)^{-\lambda_{m}(w)}+\frac{k-j}{k^{2}} w \tilde{T}(z) \\
& =\frac{j}{k} \mathscr{M}_{j-1}(z)+\frac{w}{k} \tilde{T}(z) \\
& =\frac{j}{k} \sum_{1 \leq m \leq k} h_{j-1, m}(w)(1-z)^{-\lambda_{m}(w)}-\frac{j}{k^{2}} w \tilde{T}(z)+\frac{1}{k} w \tilde{T}(z),
\end{aligned}
$$


which leads to the recurrence

$$
h_{j, m}(w)=\frac{j}{k \lambda_{m}(w)-k+j-1} h_{j-1, m}(w) \quad(1 \leq m, j \leq k),
$$

where $h_{0, m}:=w h_{k, m}$. Iterating this recurrence gives

$$
\begin{aligned}
h_{j, m}(w) & =\frac{j ! w}{\prod_{k-j+1 \leq s \leq k}\left(k \lambda_{m}(w)-s\right)} h_{k, m}(w) \\
& =\frac{j ! w(w-1)}{\left(k \lambda_{m}(w)-1\right)\left(\sum_{1 \leq s \leq k} \frac{1}{k \lambda_{m}(w)-s}\right) \prod_{k-j+1 \leq s \leq k+1}\left(k \lambda_{m}(w)-s\right)},
\end{aligned}
$$

for $1 \leq m, j \leq k$, provided that $w$ is not close to the origin. When $w \sim 0$, we have, by (22),

$$
h_{j, m}(w)=\frac{j !(w-1) \prod_{2 \leq s \leq k-j}\left(k \lambda_{m}(w)-s\right)}{k !\left(k \lambda_{m}(w)-k-1\right) \sum_{1 \leq s \leq k} \frac{1}{k \lambda_{m}(w)-s}},
$$

for $1 \leq m \leq k$ and $1 \leq j<k$.

\subsubsection{Properties of the zeros of $P_{k}$ and local expansions of $\lambda_{1}(w)$}

We gather here a few properties and expansions that will be needed later.

First from Figure 5, we see that the zeros of $P_{k}$ are distributed very regularly.

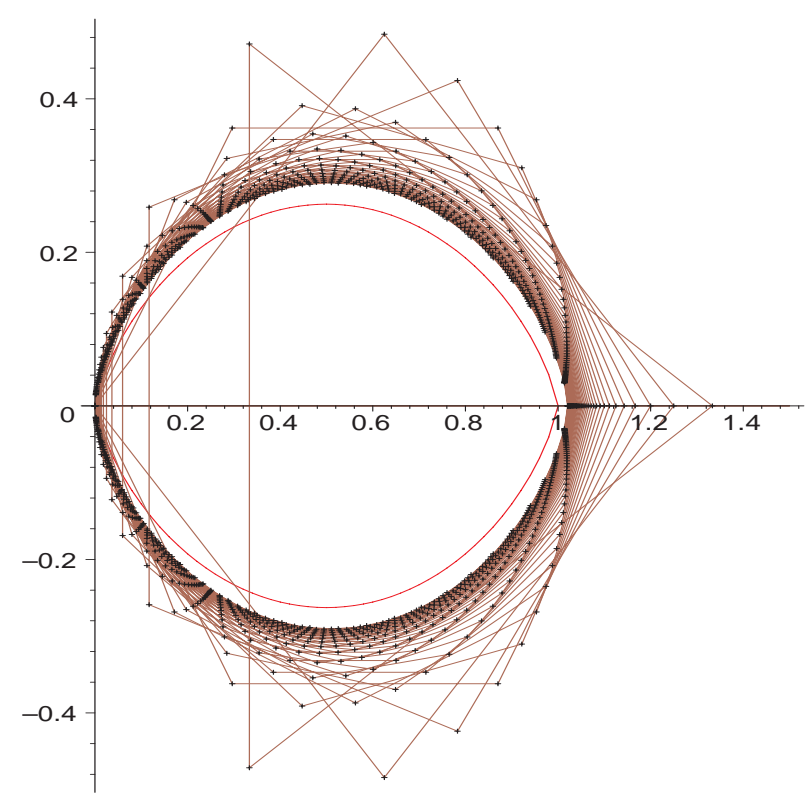

Figure 5: Distributions of the zeros of $P_{k}$ when $w=1$ for $k=3, \ldots, 50$. The limiting curve of the zeros $\left|z^{z}(z-1)^{1-z}\right|=1$ is the red innermost figure.

Most of the following properties can be proved as in [33, Ex. 6.2.4.10], [30, Annexe B], or [12]. 
Z1 All zeros are simple, and if $w \in \mathbb{C} \backslash \mathbb{R}$, then $P_{k}(\lambda)$ has no real zero.

Z2 $P_{k}$ has only one real zero for real $w$ if $k$ is odd, and two zeros if $k$ is even.

$\mathbf{Z 3}$ For real $w, \lambda_{m}(w)$ is an increasing function of $w$.

Z4 $\Re \lambda_{s}(w)=\Re \lambda_{m}(w)$ for $s \neq m$ implies that $w \in \mathbb{R}$. Note that the $\lambda_{m}(w)$ 's are themselves analytic functions, so they vary continuously on the Riemann sphere.

$\mathbf{Z 5}$ By $\mathbf{Z 3}$ and the aperiodicity of the expected profile, $\Re \lambda_{1}(w)>\Re \lambda_{m}(w)$, for $m=2, \ldots, k$ and $w \in \mathbb{C} \backslash \mathbb{R}$.

Z6 By an easy argument of contradiction, $\Re \lambda_{1}\left(r e^{i \theta}\right)<\lambda_{1}(r)$, for $r>0$ and $0<|\theta|<\pi$.

Z7 Since $\prod_{1 \leq \ell \leq k}\left(\lambda_{m}(w)-\ell / k\right)=c_{k} w$, we see that if $w \rightarrow 0$, then one of the zeros of $P_{k}$ tends to $\ell / k$; by construction, $\lambda_{1}(w) \sim 1$ as $w \sim 0$, and for the other zeros we may assume that $\lambda_{m}(w) \sim(k-m+1) / k$ as $w \sim 0$. Thus if $w>0$, then $\lambda_{m}(w)>(k-m+1) / k$.

Z8 As $k \rightarrow \infty$, all zeros approach the limiting curve $\left|z^{z}(z-1)^{1-z}\right|=|w|$, as can be proved by Stirling's formula for the Gamma function; see Figure 5.

Local expansions of $\lambda_{1}(w)$. In the next step of the proof, we also need the local expansions of $\lambda_{1}(w)$. Consider first $w \sim 0$. By writing

$$
\prod_{1 \leq \ell \leq k}\left(\theta-\frac{\ell}{k}\right)=(\theta-1) \prod_{1 \leq \ell<k}\left(\theta-1+\frac{\ell}{k}\right)=\frac{k !}{k^{k}}(\theta-1) \prod_{1 \leq \ell<k}\left(1+\frac{k}{\ell}(\theta-1)\right),
$$

we have

$$
\lambda_{1}(w)-1=\frac{w}{\prod_{1 \leq \ell<k}\left(1+\frac{k}{\ell}\left(\lambda_{1}(w)-1\right)\right)},
$$

which, by the Lagrange inversion formula (see [24, Appendix A.5]), gives the expansion as $w \sim 0$

$$
\begin{aligned}
\lambda_{1}(w)-1=w & -k H_{k-1} w^{2}+\frac{k^{2}}{2}\left(3 H_{k-1}^{2}+H_{k-1}^{(2)}\right) w^{3} \\
& -\frac{k^{3}}{3}\left(8 H_{k-1}^{3}+6 H_{k-1} H_{k-1}^{(2)}+H_{k-1}^{(3)}\right) w^{4}+\cdots
\end{aligned}
$$

Similarly, for $w \sim 1$, we obtain $\lambda_{1}(1)=(k+1) / k$ and

$$
\prod_{1 \leq \ell \leq k}\left(1+\frac{k}{\ell}\left(\lambda_{1}(w)-\frac{k+1}{k}\right)\right)-1=w-1
$$

and thus

$$
\begin{aligned}
\lambda_{1}(w)-\frac{k+1}{k}= & \frac{1}{k H_{k}}(w-1)-\frac{H_{k}^{2}-H_{k}^{(2)}}{2 k H_{k}^{3}}(w-1)^{2} \\
& +\frac{2 H_{k}^{4}-3 H_{k}^{2} H_{k}^{(2)}-H_{k} H_{k}^{(3)}+3\left(H_{k}^{(2)}\right)^{2}}{6 k H_{k}^{5}}(w-1)^{3}+\cdots
\end{aligned}
$$




\subsubsection{From $\mathscr{M}_{j}$ to $\tilde{M}_{d, j}$}

With the expressions for the coefficient-functions $h_{j, m}$ at hand, we can now estimate the coefficient of $z^{n}$ in $\tilde{M}_{d, j}$. From (17), we have, for $d \geq 1$,

$$
\left[z^{n}\right] \tilde{M}_{d, j}(z)=\left[w^{d} z^{n}\right] \mathscr{M}_{j}(z, w) .
$$

We use singularity analysis for extracting asymptotically the coefficient of $z^{n}$ in $\mathscr{M}_{j}(z, w)$, and then saddle point method for the asymptotics of the coefficient of $w^{d}$ in the resulting expression.

Singularity analysis. From (17), we have the following identity, for $d \geq 1$

$$
\left[z^{n}\right] \tilde{M}_{d, j}(z)=\left[w^{d}\right]\left(\sum_{1 \leq m \leq k} h_{j, m}(w)\left(\begin{array}{c}
\lambda_{m}(w)+n-1 \\
n
\end{array}\right)-\frac{w-(w-1) \delta_{k, j}}{k}\left(\begin{array}{c}
\frac{1}{k}+n-1 \\
n
\end{array}\right)\right) .
$$

By singularity analysis, we have, for $d \geq 1$

$$
\begin{aligned}
{\left[z^{n}\right] \tilde{M}_{d, j}(z)=} & {\left[w^{d}\right]\left(\sum_{1 \leq m \leq k} \frac{h_{j, m}(w)}{\Gamma\left(\lambda_{m}(w)\right)} n^{\lambda_{m}(w)-1}\left(1+O\left(n^{-1}\right)\right)\right)+O\left(\delta_{d, 1} n^{-1+1 / k}\right) } \\
= & \frac{1}{2 \pi i} \oint_{|w|=\rho} w^{-d-1}\left(\sum_{1 \leq m \leq k} \frac{h_{j, m}(w)}{\Gamma\left(\lambda_{m}(w)\right)} n^{\lambda_{m}(w)-1}\left(1+O\left(n^{-1}\right)\right)\right) \mathrm{d} w \\
& +O\left(\delta_{d, 1} n^{-1+1 / k}\right),
\end{aligned}
$$

where $\rho>0$ is chosen to be the saddle-point $\rho \lambda_{1}^{\prime}(\rho)=d / \log n$.

Saddle-point approximations. Now by the properties of the zeros of $P_{k}$, we deduce that

$$
\left[z^{n}\right] \tilde{M}_{d, j}(z)=\frac{1}{2 \pi i} \int_{\substack{|w|=\rho \\|\arg (w)| \leq \varepsilon}} \frac{h_{j, 1}(w)}{\Gamma\left(\lambda_{1}(w)\right)} w^{-d-1} n^{\lambda_{1}(w)-1}\left(1+O\left(n^{-\varepsilon}\right)\right) \mathrm{d} w ;
$$

see $[12,31]$ for similar details.

Consider now $d=o(\log n)$, so that the saddle-point $\rho \sim 0$. We have

$$
h_{j, 1}(w)=\frac{j}{k-1} w\left(1+O\left(H_{k}|w|\right)\right) \quad(w \sim 0) .
$$

It follows, by (27), (25) and a careful error analysis, that

$$
\left[z^{n}\right] \tilde{M}_{d, j}(z) \sim \frac{j}{k-1}\left[w^{d-1}\right] n^{w}=\frac{j}{k-1} \cdot \frac{(\log n)^{d-1}}{(d-1) !},
$$

the error term dropped being of order $O\left(k H_{k} d / \log n\right)$. Thus this expression holds uniformly for $d=o(\log n)$, and the corresponding expected value is given in (6). Note that if $\alpha=$ $d / \log n$, then $(\log n)^{d} / d ! \asymp d^{-1 / 2} n^{\alpha-\alpha \log \alpha}$. 
For larger values of $d$ (indeed for $d \rightarrow \infty$ ), we again use (27) and apply a direct saddlepoint method; the result is

$$
\left[z^{n}\right] \tilde{M}_{d, j}(z) \sim \frac{h_{j, 1}(\rho) \rho^{-d} n^{\lambda_{1}(\rho)-1}}{\Gamma\left(\lambda_{1}(\rho)\right) \sqrt{2 \pi\left(\rho \lambda_{1}^{\prime}(\rho)+\rho^{2} \lambda_{1}^{\prime \prime}(\rho)\right) \log n}},
$$

where $\rho=\rho_{n, d}>0$ solves the equation $\rho \lambda_{1}^{\prime}(\rho)=d / \log n$. This gives the corresponding expected value in (7).

\subsection{Proofs of some corollaries of Theorem 2}

Gaussian approximation (10) of Corollary 2. Since $\lambda_{1}(1)=(k+1) / k$, we see in this case that $\rho=1$,

$$
\rho \lambda_{1}^{\prime}(\rho)=\frac{1}{\sum_{1 \leq \ell \leq k} \frac{1}{\lambda_{1}(\rho)-\frac{\ell}{k}}},
$$

and $\lambda_{1}(\rho)-1 / k-\alpha \log \rho \sim 1$. The more precise asymptotic estimate (10) then follows from a straightforward analysis using (7) and the local expansion (26).

The expected height. From the estimate (7) for the expected profile, we can get an upper bound for the height. The argument is standard and as follows. Let $\mathscr{H}_{n}$ denote the height. Then

$$
\begin{aligned}
\mathbb{E}\left(\mathscr{H}_{n}\right) & =\left(\sum_{d<d_{0}}+\sum_{d \geq d_{0}}\right) \mathbb{P}\left(\mathscr{H}_{n} \geq d\right) \\
& \leq d_{0}+\sum_{d \geq d_{0}} \mathbb{P}\left(\mathscr{H}_{n} \geq d\right),
\end{aligned}
$$

for a suitably chosen $d_{0}$. So we derive an upper bound for the last sum. Let $X_{n, d}$ denote the profile (for some $j=1, \ldots, k$ ). Now

$$
\left.\mathbb{P}\left(\mathscr{H}_{n} \geq d\right) \leq \sum_{\ell \geq d} \mathbb{P}\left(X_{n, \ell}>0\right)\right) \leq \sum_{\ell \geq d} \mathbb{E}\left(X_{n, \ell}\right)
$$

Then, assuming that $d_{0}$ is an integer,

$$
\begin{aligned}
\sum_{d \geq d_{0}} \mathbb{P}\left(\mathscr{H}_{n} \geq d\right) & \leq \sum_{d \geq d_{0}} \sum_{\ell \geq d} \mathbb{E}\left(X_{n, \ell}\right) \\
& =\sum_{\ell \geq d_{0}}\left(\ell-d_{0}+1\right) \mathbb{E}\left(X_{n, \ell}\right) \\
& =\frac{1}{\left(\begin{array}{c}
\frac{1}{k}+n-1 \\
n
\end{array}\right)}\left[z^{n}\right] \sum_{\ell \geq d_{0}}\left(\ell-d_{0}+1\right)\left[w^{\ell}\right] \mathscr{M}(z, w) \\
& =\frac{1}{\left(\begin{array}{c}
\frac{1}{k}+n-1 \\
n
\end{array}\right)}\left[z^{n}\right]\left[w^{d_{0}}\right] \frac{\mathscr{M}(z, w)}{(1-1 / w)^{2}}
\end{aligned}
$$


where $\mathscr{M}=\mathscr{M}_{j}$ for some $j$. By our saddle-point analysis, the additional factor $(1-1 / w)^{2}$ in the denominator does not change the asymptotic order of the expected profile if we choose $d$ large enough (so that $\rho>1$ ), and we also have, by a similar (and simpler) analysis as above,

$$
\sum_{d \geq d_{0}} \mathbb{P}\left(\mathscr{H}_{n} \geq d\right)=O\left((\log n)^{-1 / 2} \rho^{-d_{0}} n^{\lambda_{1}(\rho)-1 / k}\right)
$$

where $\rho \lambda_{1}^{\prime}(\rho)=d_{0} / \log n$.

For the choice of $d_{0}$, we first identify the location where the exponent of $n$ in (7), which is $\lambda_{1}(\rho)-1 / k-\alpha \log \rho$, will tend to zero for the first time as $\rho$ increases. This can be obtained by solving the system of equations (12) for positive reals $\left(\alpha_{+}, v\right)$, where $v=\lambda_{1}\left(\alpha_{+}\right)$. In this way, we obtain Table 2.

For large $k$, one can show that $\alpha_{+} \sim 1 /(k \log 2)$ and $\lambda_{1}\left(\alpha_{+}\right) \sim 2$.

Now we take $d_{0}=\left\lfloor\alpha_{+} \log n-\beta_{+} \log \log n+O(1)\right\rfloor$, where

$$
\beta_{+}:=\frac{\alpha_{+}}{2\left(\lambda_{1}\left(\alpha_{+}\right)-\frac{1}{k}\right)},
$$

and then get (11). The upper bound (11) is, up to the second-order term, expected to be tight. This proves Corollary 3.

\section{Limiting distributions}

With the availability of the bivariate generating functions (2), we can proceed further and derive the limit distribution of $X_{n ; d, j}$ in the range when $d=O(1)$. The case when $d \rightarrow \infty$ is expected to be more involved; we content ourselves in this paper with the statement of the result for bounded $d$. It might be possible to further extend the limit result to higher values of $d$ by the method used in[27], but the details would be very messy.

As in the case of expected connectivity-profile, we first present our results, and the proofs are collected at the end of the section.

\subsection{Distribution of the connectivity-profile}

Theorem 3. The random variables $X_{n ; d, j}$, when normalized by their mean orders, converge in distribution to

$$
\frac{X_{n ; d, j}}{n^{1-1 / k}(\log n)^{d-1} /(d-1) !} \stackrel{d}{\longrightarrow} \Xi_{d, j},
$$

where the limit law $\Xi_{d, j}$ has the moment generating function

$$
\begin{aligned}
\mathbb{E}\left(e^{\Xi_{d, j} u}\right) & =\Gamma\left(\frac{1}{k}\right) \sum_{m \geq 0} \frac{c_{d, j, m}}{m ! \Gamma(m(1-1 / k)+1 / k)} u^{m} \\
& =\frac{\Gamma\left(\frac{1}{k}\right)}{2 \pi i} \int_{-\infty}^{(0+)} e^{\tau} \tau^{-1 / k} C_{d, j}\left(\tau^{-1+1 / k} u\right) d \tau,
\end{aligned}
$$

and $C_{d, j}(u):=1+\sum_{m \geq 1} c_{d, j, m} u^{m} / m$ ! satisfies the system of DEs

$$
(k-1) u C_{d, j}^{\prime}(u)+C_{d, j}(u)=C_{d, j}(u)^{k+1-j} C_{d, j-1}(u)^{j},
$$


for $1 \leq j \leq k$, with $C_{d, 0}=C_{d-1, k}$ and $C_{1,0}=1$. Here the symbol $\int_{-\infty}^{(0+)}$ denotes any Hankel contour starting from $-\infty$ on the real axis, encircling the origin once counter-clockwise, and returning to $-\infty$.

The proof of the theorem is given in Section 4.3. We shall indeed prove convergence of all moments, which is stronger than weak convergence; also the limit law is uniquely determined by its moment sequence. What is interesting here is that the limit laws are characterized by a system of nonlinear differential equations, bearing a similar form as the starting system of DEs (3).

Exactly solvable cases for $C_{1, j}$. Only in some special cases do we obtain explicit solutions for $C_{1, j}$

$$
\begin{aligned}
& C_{1,1}(u)=(1-u)^{-1 /(k-1)} \quad(k \geq 2), \\
& C_{1,2}(u)=\frac{e^{u /(1-u)}}{1-u} \quad(k=2),
\end{aligned}
$$

and, for $k \geq 3$,

$$
C_{1,2}(u)=\varphi(u)^{-\frac{1}{k-2}}
$$

where for $|u|<1$

$$
\begin{aligned}
\varphi(u) & :=1-\frac{k-2}{k-1} \sum_{m \geq 1}\left(\begin{array}{c}
m+\frac{2}{k-1}-1 \\
m
\end{array}\right) \frac{u^{m}}{m-\frac{k-2}{k-1}} \\
& =(1-u)^{-\frac{2}{k-1}}-2 u-\frac{2 u}{k-1} \int_{0}^{1} t^{-\frac{k-2}{k-1}}\left((1-u t)^{-\frac{2}{k-1}-1}-1\right) \mathrm{d} t .
\end{aligned}
$$

In particular, when $k=3$, we have

$$
C_{1,2}(u)=\frac{1}{1-\frac{\sqrt{u}}{2} \log \frac{1+\sqrt{u}}{1-\sqrt{u}}} .
$$

All these solutions can be easily checked. More solutions will be discussed elsewhere.

Rayleigh distribution. Note that the result (28) when $d=1$ can also be derived directly by the explicit expression (1). In particular, when $k=2$, the limit law is Rayleigh, with density $t e^{-t^{2} / 4} / 2$ for $t \geq 0$, since

$$
\sqrt{\pi} \sum_{m \geq 0} \frac{u^{m}}{\Gamma((m+1) / 2)}=\frac{1}{2} \int_{0}^{\infty} t e^{t u-t^{2} / 4} \mathrm{~d} t .
$$

See Figures 6 and 7 for simulated results. For higher values of $k$, the moment generating function of $\Xi$ is expressible in terms of the generalized hypergeometric functions.

Variance. As a corollary of theorem3, we also get an explicit asymptotic expression for the variance of of $X_{n ; d, j}$, when $d=O(1)$. 


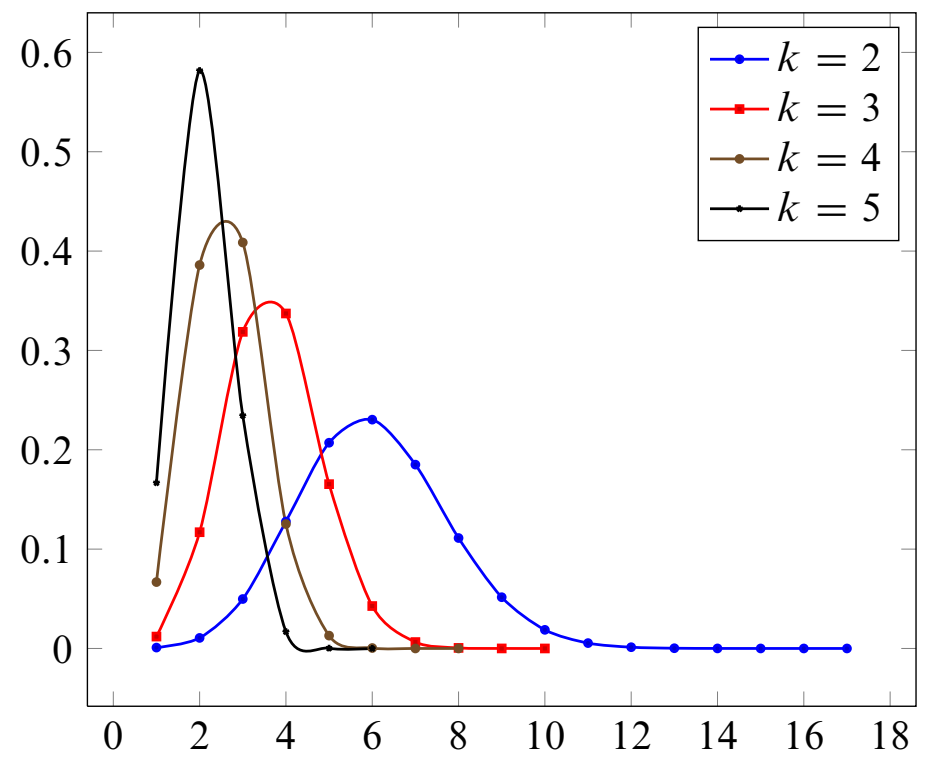

Figure 6: The profile of a single random increasing $k$-tree of size $8 \cdot 10^{7}$ for different values of $k$.

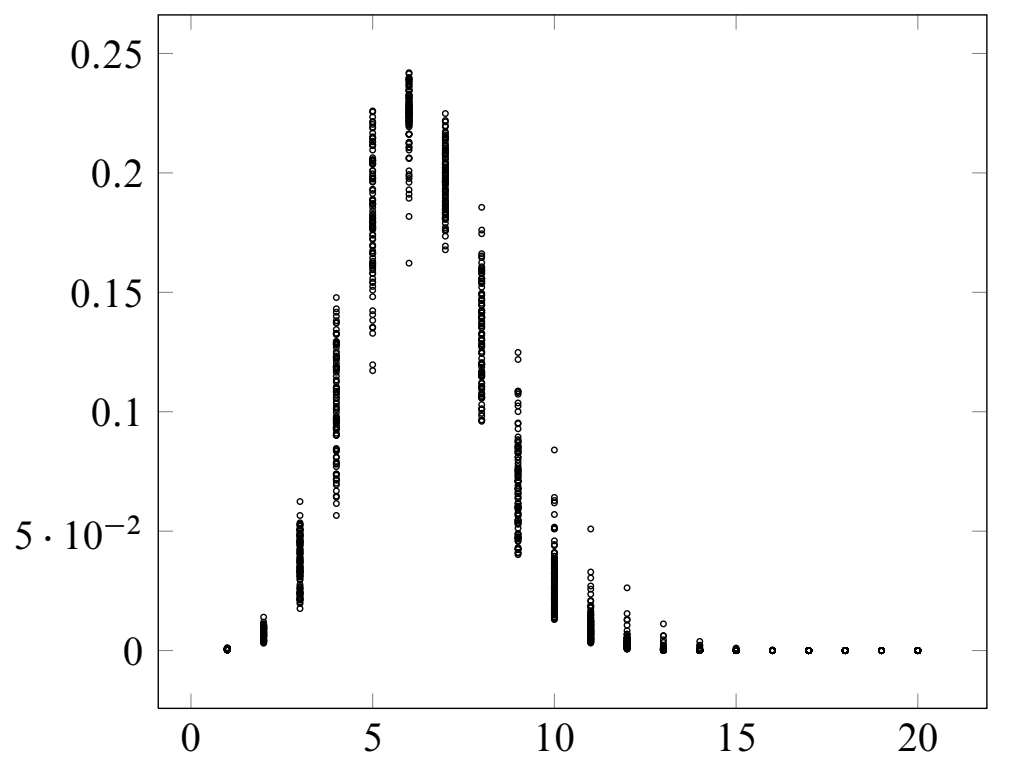

Figure 7: The profiles of 100 random increasing 2-trees of size $5 \cdot 10^{7}$. 
Corollary 5. The variance of $X_{n ; d, j}$ is asymptotic to

$$
\mathbb{V}\left(X_{n ; d, j}\right) \sim\left(\frac{\Gamma\left(\frac{1}{k}\right)}{\Gamma\left(2-\frac{1}{k}\right)} c_{d, j, 2}-\left(\frac{j \Gamma\left(\frac{1}{k}\right)}{k-1}\right)^{2}\right)\left(n^{1-1 / k} \frac{(\log n)^{d-1}}{(d-1) !}\right)^{2},
$$

for $d=O(1)$ and $1 \leq j \leq k$, where $c_{d, j, 2}$ is given below in (43).

Proof. See Section 4.3.4

In what follows, we start with the proof of Theorem 3 in the simplest case when $d=j=1$, for which $X_{n ; 1,1}$ is nothing but the root degree. Our method of proof is based on exact solution and the method of moments. Then we consider the case when $d=1$ and $1 \leq j \leq k$ for which closed-form solutions are too messy even when available; we thus use an inductive argument for the asymptotics of moments and still apply the method of moments. The proof is then easily amended for the remaining cases $d \geq 1$ and $1 \leq j \leq k$.

$$
\begin{gathered}
d=j=1 \\
\text { (root degree) }
\end{gathered} \rightarrow \begin{gathered}
d=1 \\
1 \leq j \leq k
\end{gathered} \rightarrow \begin{gathered}
d \geq 1 \\
1 \leq j \leq k
\end{gathered}
$$

\subsection{Degree distribution of the root}

Consider the special case when $d=j=1$. We state the following result for ease of reference although it is a special case of Theorem 3 when $d=j=1$.

Theorem 4. The degree $X_{n ; 1,1}$ of the root-clique in a random $k$-tree of $n+k$ vertices satisfies

$$
\frac{X_{n ; 1,1}}{n^{1-1 / k}} \stackrel{d}{\longrightarrow} \Xi_{1,1}
$$

where the limit law $\Xi_{1,1}=\Xi_{1,1}(k)$ has the moment generating function

$$
\mathbb{E}\left(e^{\Xi_{1,1} u}\right)=\frac{\Gamma\left(\frac{1}{k}\right)}{\Gamma\left(\frac{1}{k-1}\right)} \sum_{m \geq 0} \frac{\Gamma\left(m+\frac{1}{k-1}\right)}{\Gamma\left(m\left(1-\frac{1}{k}\right)+\frac{1}{k}\right) m !} u^{m} .
$$

Bivariate generating function. By (3), the generating function $F(z, u):=\tilde{T}_{1,1}(z, u)$ satisfies the differential equation

$$
\frac{\partial}{\partial z} F(z, u)=\frac{u}{k} \tilde{T}(z) F^{k}(z, u)
$$

Lemma 2. The solution to the equation (31) is given by

$$
F(z, u)=\left(1-u\left(1-Z^{1-1 / k}\right)\right)^{-1 /(k-1)}, \quad \text { with } \quad Z=1-z
$$




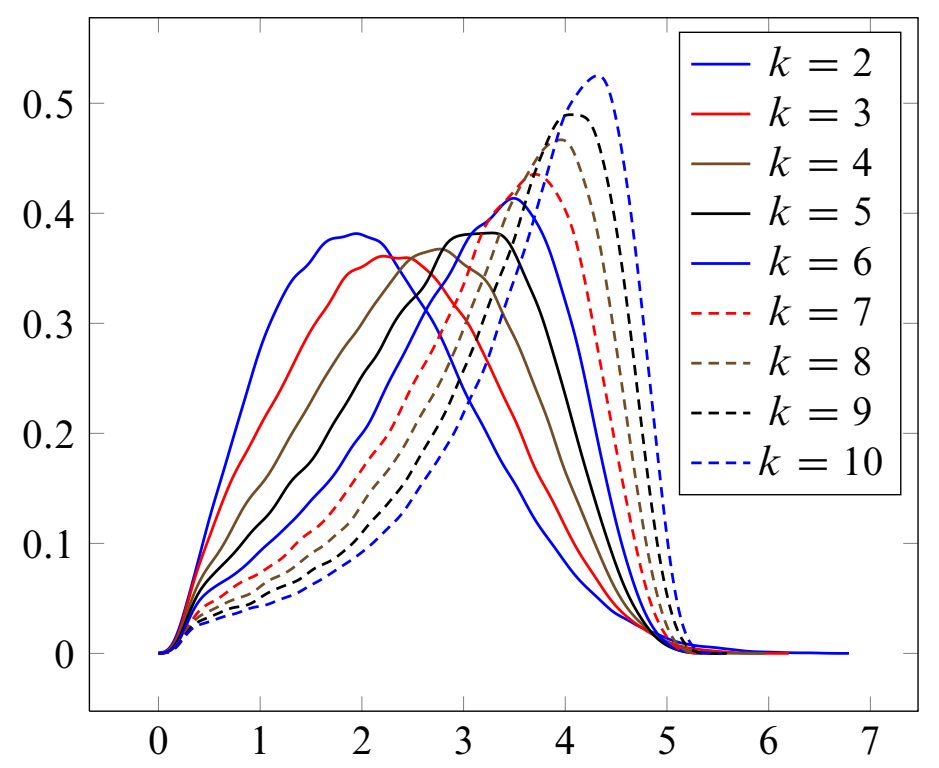

Figure 8: Distribution of the root degree of a random increasing $k$-tree for $k$ from 2 to 10.

Proof. The solution (32) is easily checked, once available; it is also similar to (1). To derive it, let

$$
F(z, u):=1+\sum_{m \geq 1} f_{m}(z) \frac{u^{m}}{m !} .
$$

Then, by (31), we see that $f_{m}$ satisfies

$$
f_{m}^{\prime}=\frac{\tilde{T}}{k} \sum_{\substack{j_{1}+\cdots+j_{k}=m-1 \\ j_{1}, \ldots, j_{k} \geq 0}} \frac{m !}{j_{1} ! \cdots j_{k} !} f_{j_{1}} \cdots f_{j_{k}} \quad(m \geq 1),
$$

with $f_{m}(0)=0$. Then we have

$$
f_{1}=\frac{1}{k-1}\left(1-Z^{1-1 / k}\right), \quad \text { and } \quad f_{2}=\frac{k}{(k-1)^{2}}\left(1-Z^{1-1 / k}\right)^{2},
$$

and, by induction,

$$
\begin{aligned}
f_{m}(z) & =\frac{\prod_{1 \leq \ell<m}(\ell k-\ell+1)}{(k-1)^{m}}\left(1-Z^{1-1 / k}\right)^{m} \\
& =\frac{\Gamma\left(m+\frac{1}{k-1}\right)}{\Gamma\left(\frac{1}{k-1}\right)}\left(1-Z^{1-1 / k}\right)^{m} \quad(m \geq 0) .
\end{aligned}
$$

From this explicit solution, (32) follows.

Corollary 6. The probability that the root-clique has degree $m$ satisfies

$$
\mathbb{P}\left(X_{n ; 1,1}=m\right) \sim \frac{\Gamma\left(m+\frac{1}{k-1}\right)}{\Gamma(m) \Gamma\left(\frac{1}{k-1}\right)} \cdot \frac{k-1}{k} n^{-1},
$$

uniformly for $1 \leq m=o\left(n^{1 / 2}\right)$. 
Proof. By (33), we have, for $m \geq 1$,

$$
\begin{aligned}
\mathbb{P}\left(X_{n ; 1,1}=m\right) & =\frac{\left[z^{n} u^{m}\right] F(z, u)}{\left[z^{n}\right](1-z)^{-1 / k}} \\
& =\frac{\Gamma\left(m+\frac{1}{k-1}\right)}{m ! \Gamma\left(\frac{1}{k-1}\right)\left(\begin{array}{c}
n+1 / k-1 \\
n
\end{array}\right)}\left[z^{n}\right]\left(1-(1-z)^{1-1 / k}\right)^{m} \\
& \sim \frac{\Gamma\left(m+\frac{1}{k-1}\right)}{m ! \Gamma\left(\frac{1}{k-1}\right)\left(\begin{array}{c}
n+1 / k-1 \\
n
\end{array}\right)} \cdot \frac{m(k-1)}{k \Gamma\left(\frac{1}{k}\right)} n^{-2+1 / k},
\end{aligned}
$$

uniformly for $m=o\left(n^{1 / 2}\right)$. Thus (34) follows.

In particular, when $m \rightarrow \infty$ and $m=o\left(n^{1 / 2}\right)$, we have

$$
\mathbb{P}\left(X_{n ; 1,1}=m\right) \sim \frac{m^{1 /(k-1)}}{k \Gamma\left(\frac{k}{k-1}\right)} n^{-1} .
$$

One can also derive more precise approximations for larger values of $m$ by a direct application of the saddle-point method. Also the dependence on $m$ is asymptotically a Beta function.

Remark. Inspired by the explicit solution (32), we can express the solution to the other $\tilde{T}_{d, j}$ as follows.

$$
\tilde{T}_{d, j}(z, u)=\left(1-\frac{k-j}{k} u^{\delta_{d, 1}} \int_{0}^{z} \tilde{T}_{d, j-1}^{j}(t, u) \mathrm{d} t\right)^{-1 /(k-j)} .
$$

Indeed, (32) also follows from this with $d=1$ and $j=1$.

In particular,

$$
\tilde{T}_{1,2}(z, u)=\left(1-\frac{k-2}{k} u \int_{0}^{z}\left(1-u\left(1-(1-t)^{1-1 / k}\right)\right)^{-2 /(k-1)} \mathrm{d} t\right)^{-1 /(k-2)} .
$$

But the expressions for higher values of $d$ or $j$ soon become messy and less useful.

Method of moments. The explicit expression (32) can be re-written as

$$
\begin{aligned}
\tilde{T}_{1,1}(z, u) & =Z^{-1 / k}\left(1-(u-1) Z^{-1+1 / k}\left(1-Z^{1-1 / k}\right)\right)^{-1 /(k-1)} \\
& =Z^{-1 / k} \sum_{m \geq 0}\left(\begin{array}{c}
\frac{1}{k-1}+m-1 \\
m
\end{array}\right)(u-1)^{m} Z^{-m(1-1 / k)}\left(1-Z^{1-1 / k}\right)^{m} .
\end{aligned}
$$

Thus all factorial moments of the random variable $X_{n ; 1,1}$ can be directly computed, and we have

$$
\begin{aligned}
\mathbb{E}\left(X_{n ; 1,1} \cdots\left(X_{n ; 1,1}-m+1\right)\right) & =\frac{\Gamma\left(m+\frac{1}{k-1}\right)}{\Gamma\left(\frac{1}{k-1}\right)} \cdot \frac{\left[z^{n}\right] Z^{-m(1-1 / k)-1 / k}\left(1-Z^{1-1 / k}\right)^{m}}{\left[z^{n}\right] \tilde{T}(z)} \\
& \sim \frac{\Gamma\left(\frac{1}{k}\right) \Gamma\left(m+\frac{1}{k-1}\right)}{\Gamma\left(\frac{1}{k-1}\right) \Gamma\left(m\left(1-\frac{1}{k}\right)+\frac{1}{k}\right)} n^{m(1-1 / k)},
\end{aligned}
$$

for $m \geq 0$. This proves the convergence of all integral moments of $X_{n ; 1,1} / n^{1-1 / k}$ to those of $\Xi$. It remains the justify that such a moment sequence uniquely determines a distribution. But 
this is easy since the moment generating function is an entire function. By the Frechet-Shohat moment convergence theorem, we conclude the proof of the theorem.

Remark. Alternatively, the limit law (30) can be proved by the following argument. Let $u:=e^{i \theta / n^{1-1 / k}}$. Then, by singularity analysis and by choosing a suitable Hankel contour $\mathcal{H}$,

$$
\begin{aligned}
& \mathbb{E}\left(e^{i X_{n ; 1,1}(n) \theta / n^{1-1 / k}}\right) \\
& =\frac{\Gamma\left(\frac{1}{k}\right) n^{1-1 / k}}{2 \pi i n} \int_{\mathcal{H}} e^{\tau}\left(1-e^{i \theta / n^{1-1 / k}}+e^{i \theta / n^{1-1 / k}}(\tau / n)^{1-1 / k}\right)^{-1 /(k-1)} \\
& \quad \times\left(1+O\left(|\tau|^{2} n^{-1}\right)\right) \mathrm{d} \tau \\
& \quad \rightarrow \frac{\Gamma\left(\frac{1}{k}\right)}{2 \pi i} \int_{-\infty}^{(0+)} e^{\tau}\left(\tau^{1-1 / k}-i \theta\right)^{-1 /(k-1)} \mathrm{d} \tau \\
& =\mathbb{E}\left(e^{\Xi_{1,1} i \theta}\right)
\end{aligned}
$$

which in the case of $k=2$ equals the characteristic function of the Rayleigh distribution. Here

the integration contour $\int_{-\infty}^{(0+)}$ starts from $-\infty$ on the real axis, encircles the origin once counterclockwise, and returns to $-\infty$. An advantage of this analytic approach is that it can be further refined and gives a convergence rate.

\subsection{Limiting distribution: $d \geq 1$ and $1 \leq j \leq k$}

For higher values of $d$ and $j$, we do not rely on explicit solutions but start from the recurrence relation derived from (3) and use instead an inductive argument, the crucial tool we need being the singularity analysis of Flajolet and Odlyzko for which we develop a notion of admissibility.

\subsubsection{Recurrence relations}

Let

$$
\tilde{T}_{d, j}(z, u)=\sum_{m \geq 0} \frac{\tilde{M}_{d, j, m}(z)}{m !}(u-1)^{m},
$$

where the $m$-th factorial moment is related to $\tilde{M}_{d, j, m}$ by

$$
\mathbb{E}\left(X_{n ; d, j}\left(X_{n ; d, j}-1\right) \cdots\left(X_{n ; d, j}-m+1\right)\right)=\frac{\left[z^{n}\right] \tilde{M}_{d, j, m}(z)}{\left[z^{n}\right] \tilde{T}(z)} .
$$

Then by (3), we have, for $d \neq 1$

$$
\frac{\tilde{M}_{d, j, m}^{\prime}(z)}{m !}=\frac{1}{k} \sum_{\substack{h_{1}+\cdots+h_{k+1-j}+i_{1}+\cdots+i_{j}=m \\ 0 \leq h_{1}, \ldots, h_{k+1-j}, i_{1}, \ldots, i_{j} \leq m}}\left(\prod_{1 \leq \ell \leq k+1-j} \frac{\tilde{M}_{d, j, h_{\ell}}(z)}{h_{\ell} !}\right)\left(\prod_{1 \leq \ell \leq j} \frac{\tilde{M}_{d, j-1, i_{\ell}}(z)}{i_{\ell} !}\right),
$$


and for $d=1$,

$$
\begin{aligned}
& \frac{\tilde{M}_{d, j, m}^{\prime}(z)}{m !}=\frac{1}{k} \sum_{\substack{h_{1}+\cdots+h_{k+1-j}+i_{1}+\cdots+i_{j}=m \\
0 \leq h_{1}, \ldots, h_{k+1-j}, i_{1}, \ldots, i_{j} \leq m}}\left(\prod_{1 \leq \ell \leq k+1-j} \frac{\tilde{M}_{d, j, h_{\ell}}(z)}{h_{\ell} !}\right)\left(\prod_{1 \leq \ell \leq j} \frac{\tilde{M}_{d, j-1, i_{\ell}}(z)}{i_{\ell} !}\right) \\
& +\frac{1}{k} \sum_{\substack{h_{1}+\cdots+h_{k+1-j}+i_{1}+\cdots+i_{j}=m-1 \\
0 \leq h_{1}, \ldots, h_{k+1-j}, i_{1}, \ldots, i_{j}<m}}\left(\prod_{\substack{1 \leq \ell \leq k+1-j \\
h_{\ell} !}} \frac{\tilde{M}_{d, j, h_{\ell}}(z)}{h_{\ell}}\right)\left(\prod_{1 \leq \ell \leq j} \frac{\tilde{M}_{d, j-1, i_{\ell}}(z)}{i_{\ell} !}\right),
\end{aligned}
$$

where the second sum will be seen to be asymptotically negligible.

\subsubsection{Flajolet-Odlyzko admissible functions}

For operational convenience, we introduce the following notion; see [24, §VI.10] for more general and thorough discussions.

Definition. A function $f$ analytic inside the unit circle is said to be $\mathscr{F} \mathscr{O}$-admissible (following Flajolet and Odlyzko [25]) if ( $i$ ) it can be analytically continued into a region of the form

$$
\mathscr{R}:=\{z:|z| \leq 1+\varepsilon,|\arg (z-1)| \geq \delta\} \backslash\{1\} \quad(\varepsilon>0 ; 0<\delta<\pi / 2),
$$

and (ii) $f$ satisfies

$$
f(z) \sim c(1-z)^{-\alpha}\left(\log \frac{1}{1-z}\right)^{\beta} \quad(c, \alpha, \beta \in \mathbb{C}),
$$

uniformly as $z \rightarrow 1$ in $\mathscr{R}$. We write simply $f \in \mathscr{F} \mathscr{O}$ or $f \in \mathscr{F} \mathscr{O}_{\alpha, \beta}$ if we want to specify the growth order.

The usefulness of such a notion resides in the asymptotic estimate

$$
\left[z^{n}\right] f(z)=\frac{c+o(1)}{\Gamma(\alpha)} n^{\alpha-1}(\log n)^{\beta},
$$

if $\alpha \neq 0,-1, \ldots$, as well as the following closure properties.

Lemma 3. $\mathscr{F} \mathscr{O}$-admissible functions are closed under addition, subtraction, multiplication, and differentiation; they are also closed under integration $\left(f \mapsto \int^{z} f\right)$ if either $\alpha>1$ or $\alpha=1, \beta>-1$.

Proof. Straightforward and details omitted. Note that if

$$
f(z) \sim \frac{1}{1-z}\left(\log \frac{1}{1-z}\right)^{\beta},
$$

then

$$
\int_{z_{0}}^{z} f(t) \mathrm{d} t \sim \begin{cases}\frac{1}{\beta+1}\left(\log \frac{1}{1-z}\right)^{\beta+1}, & \text { if } \beta>-1 ; \\ \log \log \frac{1}{1-z}, & \text { if } \beta=-1 ; \\ \text { constant, } & \text { if } \beta<-1,\end{cases}
$$


as $z \rightarrow 1$ in $\mathscr{R}$.

While one can include the powers of more iterated logarithmic functions in our definition of $\mathscr{F} \mathscr{O}$-admissible functions, still integration may fail to be closed if without additional constraint on the powers.

Lemma 4. Assume that

$$
(\theta-v) f=g \quad(v \in \mathbb{C}),
$$

in the sense of formal power series, where $\theta=(1-z) \mathbb{D}_{z}$. If $g \in \mathscr{F} \mathscr{O}_{\alpha, \beta}$, then $f \in \mathscr{F} \mathscr{O}$ provided that either $v<\alpha$ or $v=\alpha, \beta>-1$.

Proof. The solution of $f$ is given explicitly by

$$
f(z)=f(0)(1-z)^{-v}+(1-z)^{-v} \int_{0}^{z}(1-t)^{v-1} g(t) \mathrm{d} t .
$$

This provides not only an analytic continuation of $f$ into some indented disk $\mathscr{R}$ (through that of $g$ ), but also the local behavior of $f$ as $z \rightarrow 1$ in $\mathscr{R}$.

\subsubsection{Method of moments}

Assume now $d=1$ and $1 \leq j \leq k$. From (35), we see that $\tilde{M}_{1,1, m} \in \mathscr{F} \mathscr{O}$ and $\tilde{M}_{1,1, m}$ satisfies $(Z=1-z)$

$$
\tilde{M}_{1,1, m}(z) \sim\left(\begin{array}{c}
m+\frac{1}{k-1}-1 \\
m
\end{array}\right) Z^{-m(1-1 / k)-1 / k} \quad(m \geq 0),
$$

as $z \rightarrow 1$ in some indented disk $\mathscr{R}$.

We now prove by induction that $\tilde{M}_{1, j, m} \in \mathscr{F} \mathscr{O}$ and

$$
\tilde{M}_{1, j, m}(z) \sim c_{1, j, m} Z^{-m(1-1 / k)-1 / k} \quad(m \geq 0),
$$

for some explicitly computable $c_{1, j, m}$ that will be specified later. By (9), we have $c_{1, j, 0}=1$ and $c_{1, j, 1}=j /(k-1)$. Assume now $m \geq 2$. First of all, $\tilde{M}_{1, j, m}$ satisfies, by (37), the Cauchy-Euler differential equation

$$
\left(\theta-\frac{k+1-j}{k}\right) \tilde{M}_{1, j, m}(z)=Q_{1, j, m}(z),
$$

where

$$
\begin{aligned}
\frac{\tilde{Q}_{1, j, m}^{\prime}(z)}{m !} & =\frac{Z}{k} \sum_{\substack{h_{1}+\cdots+h_{k+1-j+i_{1}+\cdots+i_{j}=m} \\
0 \leq h_{1}, \ldots, h_{k+1}<j<m \\
0 \leq i_{1}, \ldots, i_{j} \leq m}}\left(\prod_{1 \leq \ell \leq k+1-j} \frac{\tilde{M}_{1, j, h_{\ell}}(z)}{h_{\ell} !}\right)\left(\prod_{1 \leq \ell \leq j} \frac{\tilde{M}_{1, j-1, i_{\ell}}(z)}{i_{\ell} !}\right) \\
& +\frac{Z}{k} \sum_{\substack{h_{1}+\cdots+h_{k+1-j}+i_{1}+\cdots+i_{j}=m-1 \\
0 \leq h_{1}, \ldots, h_{k+1-j}, i_{1}, \ldots, i_{j}<m}}^{\substack{h_{1 \leq \ell \leq k+1-j} \\
h_{\ell} !}}\left(\prod_{1 \leq \ell \leq j} \frac{\tilde{M}_{1, j, h_{\ell}}(z)}{h_{\ell} !}\right)\left(\tilde{M}_{1, j-1, i_{\ell}(z)}\right) .
\end{aligned}
$$


By Lemma 3 and induction, we have $\tilde{Q}_{1, j, m} \in \mathscr{F} \mathscr{O}$ and

$$
\tilde{Q}_{1, j, m}(z) \sim c_{1, j, m}^{\prime} Z^{-m(1-1 / k)-1 / k},
$$

where

$$
c_{1, j, m}^{\prime}=\frac{m !}{k} \sum_{\substack{h_{1}+\cdots+h_{k+1-j+i_{1}+\cdots+i_{j}=m} \\ 0 \leq h_{\ell}<m \\ 0 \leq i_{\ell} \leq m}}\left(\prod_{1 \leq \ell \leq k+1-j} \frac{c_{1, j, h_{\ell}}}{h_{\ell} !}\right)\left(\prod_{1 \leq \ell \leq j} \frac{c_{1, j-1, i_{\ell}}}{i_{\ell} !}\right) .
$$

By Lemma $4, \tilde{M}_{1, j, m} \in \mathscr{F} \mathscr{O}$. Since

$$
\tilde{M}_{d, j, m}(z)=(1-z)^{-(k+1-j) / k} \int_{0}^{z}(1-t)^{(j-1) / k} \tilde{Q}_{d, j, m}(t) \mathrm{d} t,
$$

if we choose $c_{1, j, m}$ so that they satisfy the recurrence

$$
\begin{gathered}
c_{1, j, m}=\frac{1}{m(1-1 / k)-1+j / k} \cdot \frac{1}{k} \sum_{\substack{h_{1}+\cdots+h_{k+1-j+i_{1}+\cdots+i_{j}=m} 0 \leq h_{\ell}<m \\
0 \leq i_{\ell} \leq m}}\left(\begin{array}{c}
m \\
h_{1}, \ldots, h_{k+1-j}, i_{1}, \ldots, i_{j}
\end{array}\right) \\
\times c_{1, j, h_{1}} \cdots c_{1, j, h_{k+1-j}} c_{1, j-1, i_{1}} \cdots c_{1, d, j-1, i_{j}},
\end{gathered}
$$

then (38) follows by induction. The recurrence can be rewritten, by rearranging terms, as

$$
(m(k-1)+1) \frac{c_{1, j, m}}{m !}=\sum_{\substack{h_{1}+\cdots+h_{k+1-j}+i_{1}+\cdots+i_{j}=m \\ 0 \leq h_{\ell}, i_{\ell} \leq m}}\left(\prod_{1 \leq \ell \leq k+1-j} \frac{c_{1, j, h_{\ell}}}{h_{\ell} !}\right)\left(\prod_{1 \leq \ell \leq j} \frac{c_{1, j-1, i_{\ell}}}{i_{\ell} !}\right) .
$$

In terms of the generating function $C_{d, j}(u):=\sum_{m \geq 0} c_{d, j, m} u^{m} / m$ !, we have the system of DEs

$$
(k-1) u C_{1, j}^{\prime}(u)+C_{1, j}(u)=C_{1, j}(u)^{k+1-j} C_{1, j-1}(u)^{j} \quad(1 \leq j \leq k),
$$

with $C_{1,0}(u)=1$ and $C_{1, j}(0)=1$. The proof of the convergence in distribution of $X_{n ; 1, j} / n^{1-1 / k}$ to $\Xi_{1, j}$ is complete if we prove the unique determination of the moment sequence for which it suffices to prove that the functions $C_{d, j}$ have finite radius of convergence, which will be given below for general $d$.

The same argument used above applies mutatis mutandis to $X_{n ; d, j}$ for $d \geq 2$ and we deduce (28). We omit the messy details.

This gives, up to the part of unique determination of the limit law (proved below), the convergence in distribution of $X_{n ; d, j}$ (normalized by the order of its mean) for $d=O(1)$ and $1 \leq j \leq k$.

Justification of the unique determination of the limit law. It suffices for that purpose to prove that

$$
\frac{c_{d, j, m}}{m !} \leq A^{m}\left(\begin{array}{c}
\varepsilon+m-1 \\
m
\end{array}\right),
$$




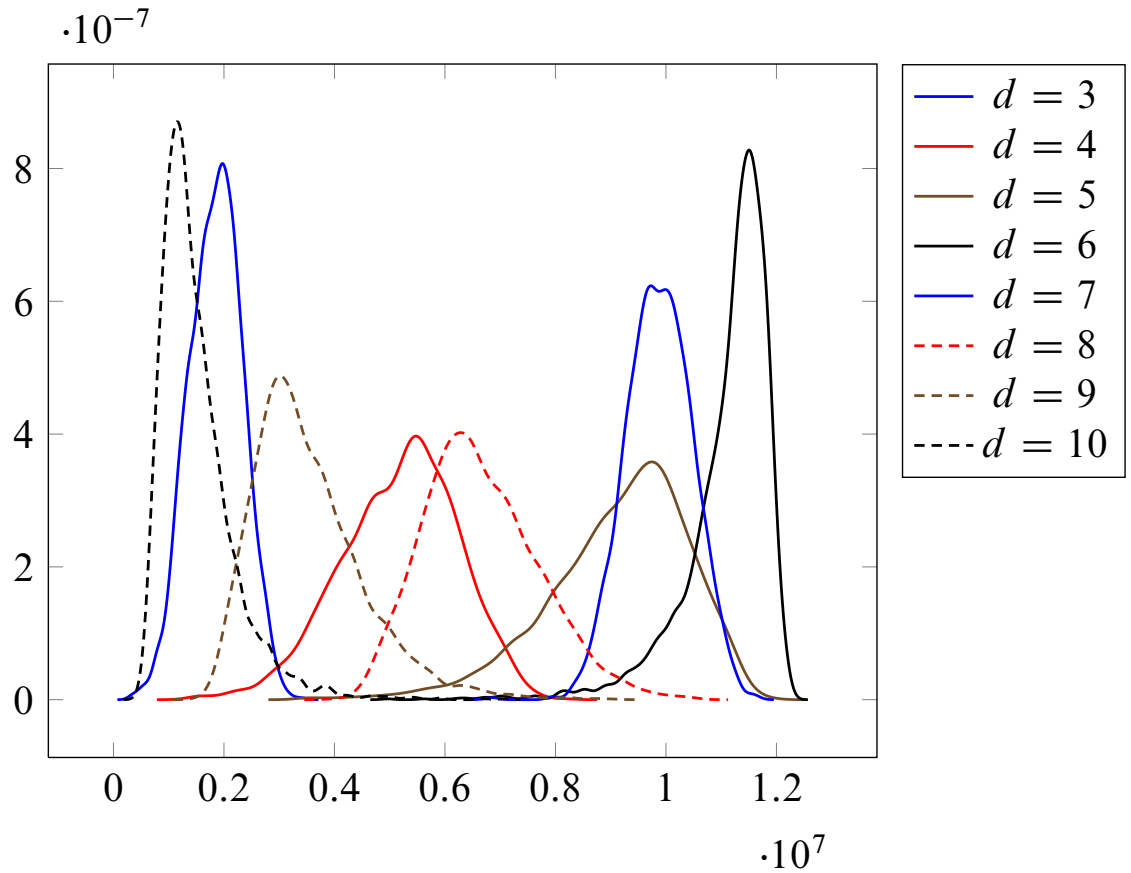

Figure 9: Distributions of the numbers of vertices at distance $d$ from the root, for $d$ from 2 to 10, as calculated from 3000 random increasing 2-trees of size $5 \cdot 10^{7}$.

for all $m \geq 0$ and $1 \leq j \leq k$, where $A>1$ is sufficiently large and $\varepsilon>0$ is sufficiently small. To prove this uniform upper bound, we start from the recurrence

$$
\frac{c_{d, j, m}}{m !}=\frac{1}{m(k-1)-k+j} \sum_{\substack{h_{1}+\cdots+h_{k+1-j+i_{1}+\cdots+i_{j}=m} \\ 0 \leq h_{\ell}<m \\ 0 \leq i_{\ell} \leq m}}\left(\prod_{1 \leq \ell \leq k+1-j} \frac{c_{d, j, h_{\ell}}}{h_{\ell} !}\right)\left(\prod_{1 \leq \ell \leq j} \frac{c_{d, j-1, i_{\ell}}}{i_{\ell} !}\right) .
$$

Then by induction

$$
\begin{aligned}
\frac{c_{d, j, m}}{m !} \leq \frac{A^{m}}{m(k-1)-k+j} \sum_{\substack{h_{1}+\cdots+h_{k+1-j+i_{1}+\cdots+i_{j}=m} \\
0 \leq h_{\ell}<m \\
0 \leq i_{\ell} \leq m}}\left(\prod_{1 \leq \ell \leq k+1-j}\left(\begin{array}{c}
\varepsilon+h_{\ell}-1 \\
h_{\ell}
\end{array}\right)\right) \\
\times\left(\prod_{1 \leq \ell \leq j}\left(\begin{array}{c}
\varepsilon+i_{\ell}-1 \\
i_{\ell}
\end{array}\right)\right) \\
\leq \frac{A^{m}}{m(k-1)-k+j}\left(\left[z^{m}\right](1-z)^{-(k+1) \varepsilon}-(k+1-j)\left(\begin{array}{c}
\varepsilon+m-1 \\
m
\end{array}\right)\right) \\
=\frac{A^{m}}{m(k-1)-k+j}\left(\left(\begin{array}{c}
(k+1) \varepsilon+m-1 \\
m
\end{array}\right)-(k+1-j)\left(\begin{array}{c}
\varepsilon+m-1 \\
m
\end{array}\right)\right),
\end{aligned}
$$


and we need the last expression to be less than or equal to $A^{m}\left(\begin{array}{c}\varepsilon+m-1 \\ m\end{array}\right)$, or equivalently

$$
\left(\begin{array}{c}
(k+1) \varepsilon+m-1 \\
m
\end{array}\right) \leq m(k-1)\left(\begin{array}{c}
\varepsilon+m-1 \\
m
\end{array}\right) \text {. }
$$

We first choose $A$ large enough so that (39) holds for $m \leq m_{0}$; then we choose $\varepsilon$ small enough so that (40) holds for $m>m_{0}$ (always possible since the left-hand side $\asymp m^{-1+(k+1) \varepsilon}$ and the right-hand side $\asymp m^{\varepsilon}$ for large $m$ ). This completes the proof of (39) and thus Theorem 3 .

\subsubsection{Variance}

We now examine the variance and compute more explicitly the coefficients $c_{d, j, 2}$, which, by (29), satisfy the recurrence

$$
c_{d, j, 2}=\frac{j}{k+j-2} c_{d, j-1,2}+\frac{j(j-1+j k(k-1))}{(k-1)^{2}(k+j-2)} .
$$

Iterating this recurrence with respect to $j$ yields

$$
c_{d, j, 2}=\frac{\Gamma(k-1) \Gamma(j+1)}{\Gamma(k+j-1)} c_{d, 0,2}+\frac{j(k-1+j+j k(k-1))}{k(k-1)^{2}} .
$$

If $d=1$, then $c_{1,0,2}=c_{0, k, 2}=0$, so that

$$
c_{1, j, 2}=\frac{j(k-1+j+j k(k-1))}{k(k-1)^{2}} .
$$

Assume now $d \geq 2$. Since $c_{d, 0,2}=c_{d-1, k, 2}$, we obtain, by substituting $j=k$ into (41),

$$
c_{d, 0,2}=c_{d-1, k, 2}=\varrho_{k} c_{d-1,0,2}+\frac{k^{3}-(k-1)^{2}}{(k-1)^{2}},
$$

where

$$
\varrho_{k}:=\frac{\Gamma(k-1) \Gamma(k+1)}{\Gamma(2 k-1)} .
$$

By iterating the recurrence (42) and by using the relation (see (34))

$$
c_{2,0,2}=c_{1, k, 2}=\frac{2}{(k-1) \Gamma(1 / k)}
$$

we have

$$
\begin{aligned}
c_{d, 0,2} & =\frac{k^{3}-(k-1)^{2}}{(k-1)^{2}} \cdot \frac{1-\varrho_{k}^{d-2}}{1-\varrho_{k}}+c_{2,0,2} \varrho_{k}^{d-2} \\
& =\frac{k^{3}-(k-1)^{2}}{(k-1)^{2}} \cdot \frac{1-\varrho_{k}^{d-2}}{1-\varrho_{k}}+\frac{2 \varrho_{k}^{d-2}}{(k-1) \Gamma(1 / k)},
\end{aligned}
$$

for $k, d \geq 2$. In particular, since $\varrho_{2}=1$, the right-hand side is equal to

$$
7(d-2)+\frac{2}{\sqrt{\pi}}
$$


Thus we obtain for $k=2$

$$
c_{d, j, 2}=7(d-2)+\frac{2}{\sqrt{\pi}}+\frac{j(3 j+1)}{2} \quad(j=1,2)
$$

and for $k \geq 3$

$$
\begin{gathered}
c_{d, j, 2}=\frac{\Gamma(k-1) \Gamma(j+1)}{\Gamma(k+j-1)(k-1)}\left(\frac{k^{3}-(k-1)^{2}}{k-1} \cdot \frac{1-\varrho_{k}^{d-2}}{1-\varrho_{k}}+\frac{2 \varrho_{k}^{d-2}}{\Gamma(1 / k)}\right) \\
+\frac{j(k-1+j+j k(k-1))}{k(k-1)^{2}} \quad(1 \leq j \leq k) .
\end{gathered}
$$

Note that if we interpret $\left(1-x^{d}\right) /(1-x)$ as $d$ when $d=1$, then (43) also holds for $k=2$.

This proves Corollary (5).

The variance for higher values of $d$ is more difficult and is expected to exhibit the same bimodal behavior as that of, say random binary search trees; see [21] and Figure 10.

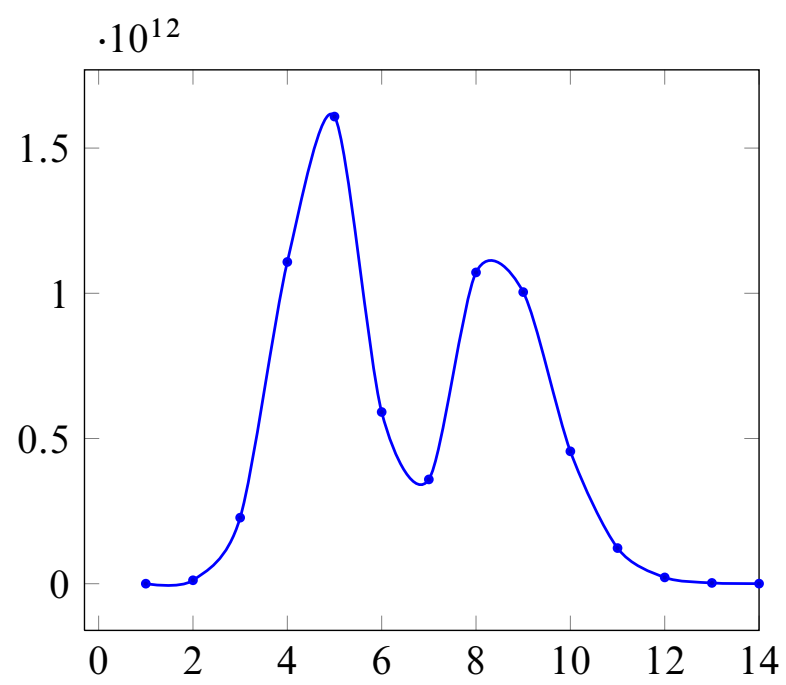

Figure 10: The variance of the connectivity-profile, calculated on 3000 random increasing 2-trees of size $5 \cdot 10^{7}$.

\section{Generation of random $k$-trees}

We conclude this paper by briefly discussing the large-scale simulations of random $k$-trees we did for the generation of the diverse figures in this paper, which may also be useful for other purposes $^{2}$.

The generation of random $k$-trees and the corresponding calculation of the profiles can easily be performed by a linear-time iterative algorithm. The iterative nature of the algorithm means that a representation of the whole graph needs to be stored in memory until the generation is finished. A naive implementation of this idea then requires that we keep enough

\footnotetext{
${ }^{2}$ The corresponding codes can be found in the first author's homepage.
} 
information to reconstruct the graph (for example, its tree representation); however, the maximum attainable size of the generated graphs is proportional to the amount of RAM available, which for $8 \cdot 10^{7}$ nodes needs about $2 \mathrm{~GB}$ of RAM.

The space complexity of the generation can be largely reduced by only keeping track of the number of nodes at distance $d$ to the root and with $i$ ancestors also at distance $d$ from the root. Such information is not only easily updated but also can be used to select a parent node when a node is generated. We thus require a storage of order $k \mathcal{H}_{n}$ when generating a $k$-tree of size $n$, which, according to Corollary 3 is $O(\log n)$.

Another way to reach a logarithmic space complexity would be to use a linear-complexity recursive algorithm [49], but its implementation is more involved.

\section{Acknowledgements}

We thank Hua-Huai Chern and Olivier Bodini for helpful comments.

\section{References}

[1] J. S. Andrade, Jr., H. J. Herrmann, R. F. S. Andrade, and L. R. da Silva. Apollonian networks: Simultaneously scale-free, small world, Euclidean, space filling, and with matching graphs. Phys. Rev. Lett., 94(1):018702 (4 pages), 2005.

[2] S. Arnborg, D. G. Corneil, and A. Proskurowski. Complexity of finding embeddings in a $k$-tree. SIAM J. Algebraic Discrete Methods, 8(2):277-284, 1987.

[3] S. Arya, M. J. Golin, and K. Mehlhorn. On the expected depth of random circuits. Combin. Probab. Comput., 8(03):209-228, 1999.

[4] A.-L. Barabási and R. Albert. Emergence of scaling in random networks. Science, 286(5439):509-512, 1999.

[5] L. W. Beineke and R. E. Pippert. The number of labeled $k$-dimensional trees. J. Combin. Theory, 6(2):200-205, 1969.

[6] F. Bergeron, P. Flajolet, and B. Salvy. Varieties of increasing trees. In $C A A P$ '92, pages 24-48. 1992.

[7] A. T. Berztiss. Depth-first $k$-trees and critical path analysis. Acta Inform., 13(4):325-346, 1980.

[8] S. Boccaletti, V. Latora, Y. Moreno, M. Chavez, and D.-U. Hwang. Complex networks: Structure and dynamics. Phys. Rep., 424(4-5):175-308, 2006.

[9] B. Bollobás, O. Riordan, J. Spencer, and G. Tusnády. The degree sequence of a scale-free random graph process. Random Structures Algorithms, 18(3):279-290, 2001.

[10] B. Chauvin, T. Klein, J.-F. Marckert, and A. Rouault. Martingales and profile of binary search trees. Electron. J. Probab., 10:420-435, 2005. 
[11] H.-H. Chern and H.-K. Hwang. Phase changes in random $m$-ary search trees and generalized quicksort. Random Structures Algorithms, 19(3-4):316-358, 2001.

[12] H.-H. Chern and H.-K. Hwang. Transitional behaviors of the average cost of Quicksort with median-of- $(2 t+1)$. Algorithmica, 29(1-2):44-69, 2001.

[13] H.-H. Chern, H.-K. Hwang, and T.-H. Tsai. An asymptotic theory for Cauchy-Euler differential equations with applications to the analysis of algorithms. J. Algorithms, 44(1):177-225, 2002.

[14] A. Darrasse, H.-K. Hwang, O. Bodini, and M. Soria. The connectivity-profile of random increasing $k$-trees. In Proceedings of the Seventh Workshop on Analytic Algorithmics and Combinatorics (ANALCO), pages 99-106. SIAM, 2010.

[15] A. Darrasse and M. Soria. Limiting distribution for distances in $k$-trees. In IWOCA 2009, Lecture Notes in Computer Science, 5874. Springer-Verlag, 2009.

[16] A. Darrasse and M. Soria. A unifying structural approach to the analysis of parameters in $k$-trees. Submitted, 2011.

[17] L. Devroye and S. Janson. Long and short paths in uniform random recursive dags. Arkiv för Matematik, 49(1):61-77, 2011.

[18] M. Drmota. Random Trees. Springer Vienna, 2009.

[19] M. Drmota and B. Gittenberger. On the profile of random trees. Random Structures Algorithms, 10(4):421-451, 1997.

[20] M. Drmota, S. Janson, and R. Neininger. A functional limit theorem for the profile of search trees. Ann. Appl. Proba., 18(1):288-333, 2008.

[21] Michael Drmota and Hsien-Kuei Hwang. Bimodality and phase transitions in the profile variance of random binary search trees. SIAM J. Discrete Math., 19(1):19-45 (electronic), 2005.

[22] R. Durrett. Random Graph Dynamics. Number 20 in Cambridge Series in Statistical and Probabilistic Mathematics. Cambridge University Press, 2006.

[23] M. L. Fisher. Optimal solution of vehicle routing problems using minimum k-trees. Oper. Res., 42(4):626-642, 1994.

[24] F. Flajolet and R. Sedgewick. Analytic Combinatorics. Cambridge University Press, 2009.

[25] P. Flajolet and A. Odlyzko. Singularity analysis of generating functions. SIAM J. Discrete Math., 3(2):216-240, 1990.

[26] A. M. Frieze and C. E. Tsourakakis. High degree vertices, eigenvalues and diameter of random Apollonian networks. CoRR, abs/1104.5259, 2011.

[27] M. Fuchs, H.-K. Hwang, and R. Neininger. Profiles of random trees: Limit theorems for random recursive trees and binary search trees. Algorithmica, 46(3-4):367-407, 2006. 
[28] Y. Gao. The degree distribution of random $k$-trees. Theor. Comput. Sci., 410(8-10):688695, 2009.

[29] D. H. Greene. Labelled Formal Languages and Their Uses. Ph.D. Thesis, Stanford University Stanford, CA, USA, 1983.

[30] P. Hennequin. Analyse en moyenne d'algorithme, tri rapide et arbres de recherche. Thèse, LIX, École polytechnique, 1991.

[31] H-K. Hwang. Asymptotic expansions for the Stirling numbers of the first kind. J. Combin. Theory Ser. A, 71(2):343-351, 1995.

[32] H.-K. Hwang. Profiles of random trees: Plane-oriented recursive trees. Random Structures Algorithms, 30(3):380-413, 2007.

[33] D. E. Knuth. The Art of Computer Programming Volumes 3. Addison-Wesley Longman Publishing Co., Inc. Boston, MA, USA, second edition, 1998.

[34] G. Labelle, C. Lamathe, and P. Leroux. Labelled and unlabelled enumeration of $k$-gonal 2-trees. J. Combin. Theory Ser. A, 106(2):193-219, 2004.

[35] G. Lin. An improved approximation algorithm for multicast $k$-tree routing. J. Comb. Optim., 9(4):349-356, 2005.

[36] J.-F. Marckert and M. Albenque. Some families of increasing planar maps. Electron. J. Probab., 13:1624-1671, 2008.

[37] C. Martinhon, A. Lucena, and N. Maculan. Stronger $K$-tree relaxations for the vehicle routing problem. European J. Oper. Res., 158(1):56-71, 2004.

[38] A. Meir and J. W. Moon. On the altitude of nodes in random trees. Canad. J. Math., 30:997-1015, 1978.

[39] B. Morcrette. Combinatoire analytique et modèles d'urnes. Report, Master Parisien de Recherche en Informatique, 2010.

[40] L. Newelski and A. Rosłanowski. The ideal determined by the unsymmetric game. Proc. Amer. Math. Soc., 117(3):823-831, 1993.

[41] M. E. J. Newman. The structure and function of complex networks. SIAM Rev., 45(2):167-256, 2003.

[42] E. M. Palmer and R. C. Read. On the number of plane 2-trees. J. London Math. Soc. (2), 6(4):583-592, 1973.

[43] A. Panholzer and G. Seitz. Ordered increasing $k$-trees: Introduction and analysis of a preferential attachment network model. In M. Drmota and B. Gittenberger, editors, AofA'10, pages 549-564. DMTCS, 2010.

[44] G. Park, H.-K. Hwang, P. Nicodème, and W. Szpankowski. Profiles of tries. SIAM J. Comput., 38(5):1821-1880, 2009. 
[45] I. Pávó. Generation of the $k$-trees of a graph. Acta Cybernet., 1:57-68, 1971.

[46] A. Proskurowski. $k$-trees: representation and distances. In Congr. Numer., volume 29, pages 785-794. Utilitas Mathematica, 1980.

[47] A. Rényi. On the number of endpoints of a $k$-tree. Studia Sci. Math. Hungar., 5:5-10, 1970.

[48] D. J. Rose. On simple characterizations of $k$-trees. Discrete Math., 7(3-4):317-322, 1974.

[49] O. Roussel and M. Soria. Boltzmann sampling of ordered structures. Electronic Notes in Discrete Mathematics, 35:305-310, 2009.

[50] W. R. Stevens. Traceroute program. In TCP/IP Illustrated, Volume 1: The Protocols, chapter 8. Addison-Wesley Professional, 1994.

[51] F. Viger, B. Augustin, X. Cuvellier, C. Magnien, M. Latapy, T. Friedman, and R. Teixeira. Detection, understanding, and prevention of traceroute measurement artifacts. Comput. Networks, 52(5):998-1018, 2008.

[52] S. Win. On a connection between the existence of $k$-trees and the toughness of a graph. Graphs Combin., 5(2):201-205, 1989.

[53] Z. Zhang, L. Chen, S. Zhou, L. Fang, J. Guan, and T. Zou. Analytical solution of average path length for apollonian networks. Physical Review E, 77(1):017102, 2008.

[54] Z. Zhang, L. Rong, and F. Comellas. High-dimensional random Apollonian networks. Phys. A, 364:610-618, 2006. 